\title{
Imaging and functional characterization of crop root systems using spectroscopic electrical impedance measurements
}

\author{
Maximilian Weigand (D) Andreas Kemna
}

Received: 8 June 2018 / Accepted: 31 October 2018 / Published online: 22 November 2018

(C) The Author(s) 2018

\begin{abstract}
Background and aims Non- or minimally invasive methods are urgently needed to characterize and monitor crop root systems to foster progress in phenotyping and general system understanding. Electrical methods have come into focus due to their unique sensitivity to various structural and functional root characteristics. The aim of this study is to highlight imaging capabilities of these methods with regard to crop root systems and to investigate changes in electrical signals caused by physiological reactions.

Methods Spectral electrical impedance tomography (sEIT) and electrical impedance spectroscopy (EIS) were used in three laboratory experiments to characterize oilseed root systems embedded in nutrient solution. Two experiments imaged the root extension with sEIT, including one experiment monitoring a nutrient stress situation. In the third experiment
\end{abstract}

Responsible Editor: W Richard Whalley.

Electronic supplementary material The online version of this article (https://doi.org/10.1007/s11104-018-3867-3) contains supplementary material, which is available to authorized users.

M. Weigand $(\bowtie) \cdot$ A. Kemna

Department of Geophysics, Institute of Geosciences and

Meteorology, University of Bonn,

Bonn, Germany

e-mail: mweigand@geo.uni-bonn.de

A. Kemna

e-mail: kemna@geo.uni-bonn.de electrical signatures were observed over the diurnal cycle using EIS.

Results Root system extension was imaged using sEIT under static conditions. During continuous nutrient deprivation, electrical polarization signals decreased steadily. Systematic changes were observed over the diurnal cycle, indicating further sensitivity to associated physiological processes. Spectral parameters suggest polarization processes at the $\mu \mathrm{m}$ scale.

Conclusions Electrical imaging methods are able to non-invasively characterize crop root systems in controlled laboratory conditions, thereby offering links to root structure and function. The methods have the potential to be upscaled to the field scale.

Keywords SIP · EIS · EIT · Root systems · Laboratory $\cdot$ Diurnal cycle

\section{Introduction}

Root systems play a key role in all kinds of ecosystems and are an essential part in the human food production chain (e.g., White et al. 2013, and references therein). In many ways they form the primary connection between the subsurface and the atmosphere: they are an essential part in the global hydraulic circulatory system by taking up and transpiring large amounts of water (e.g., Tinker and Nye 2000). These, and many other functions, warrant a continuous and thorough investigation of root system development 
and functionality. Linked to this is a constant requirement to optimize existing measurement methods and adapt new, preferably non-invasive ones to the field of root characterization.

Various characterization methods have been proposed at the laboratory scale (for recent overviews, see Mancuso 2012; Roose et al. 2016; Liu et al. 2017). Standing out among these are the ComputedTomography (CT) technique (e.g., Gregory et al. 2003; Metzner et al. 2015) and the Nuclear Magnetic Resonance/Magnetic Resonance Imaging (NMR/MRI) techniques (e.g., Bačić and Ratković 1987; Metzner et al. 2015), which are actively used to infer structural and physiological information on the rhizosphere. Neutron radiography has also been successfully used to image live root segments and their surrounding environment (e.g., Moradi et al. 2009; Carminati et al. 2010). However, these methods are still limited to small sample sizes and laboratory conditions (e.g., Thomas et al. 2016), and are subject to high application costs.

In this context, electrical methods emerge as an interesting alternative due to their applicability on both laboratory and field scales, and the relatively low operation costs. These methods, long established in the near-surface geophysics community, capture electrical material properties of the subsurface, or laboratory samples, which can then be related to geophysical properties such as temperature (e.g., Sen and Goode 1992), salinity (e.g., Rhoades et al. 1989), porosity/soil water content (e.g., Archie 1942; Oberdörster et al. 2010; Whalley et al. 2017), clay content (e.g., Breede et al. 2012), and even certain root parameters (e.g., Chloupek 1972; Dalton 1995; Cseresnyés et al. 2013; $\mathrm{Wu}$ et al. 2017, and references therein). It has also been recognized that electrical methods can not only be used to characterize structural, but also functional properties of root systems (e.g., Dalton 1995; Cseresnyés et al. 2014, 2016; Weigand and Kemna 2017).

Common to these methods is the injection of an electric current into the region of interest, and the subsequent measurement of the resulting electric potential difference at two locations. This process can alternatively be conducted with two or four electrodes. Four-electrode measurements are much less sensitive to electrode contact resistances (e.g., Barsoukov and Macdonald 2005) and thus favorable for bio-geophysical applications in which significant contact-resistances are common. Also, in addition to measuring the ohmic, in-phase conduction values, polarization effects in various manifestations are increasingly measured (e.g., Summer 1976; Binley and Kemna 2005; Kemna et al. 2012; Dentith and Mudge 2014). These polarization measurements provide additional, complementary information on the target object (or region), thereby reducing ambiguities in the interpretation of electrical data (e.g., Everett 2013).

While some studies measure the electrical capacitance between the injection electrodes, others measure the electrical impedance. The electrical impedance is a complex electrical quantity that comprises the inphase, ohmic conduction component as the real part and the electrical polarization in form of an imaginary part (e.g., Barsoukov and Macdonald 2005). While basically measuring the same underlying physical properties, capacitance and impedance measurements are sometimes hard to compare due to their underlying assumptions on the electrical structure of the measurement sample. Polarization effects are usually frequency dependent and are typically measured starting from $\mathrm{mHz}$ to a few tens of $\mathrm{kHz}$ (e.g., Barsoukov and Macdonald 2005).

In near-surface geophysics the measurement of polarization effects is referred to as induced polarization (IP) measurements (due to the fact that the polarization is induced, i.e., provoked, by the injected current), or complex resistivity measurements (e.g., Kemna et al. 2012). The IP effect manifests as a timeshift of the output signal (measured voltage) relative to the input current due to capacitive effects in the sample or subsurface. Spectral measurements are referred to as spectral induced polarization (SIP) or electrical impedance spectroscopy (EIS).

If measurements are conducted over heterogeneous resistivity distributions, it is common to utilize tomographic inversion algorithms to infer spatial $2 \mathrm{D}$ or 3D distributions of (complex) electrical resistivities or conductivities (e.g., LaBrecque et al. 1996; Kemna 2000; Günther et al. 2006; De Donno and Cardarelli 2014). This requires a large number of four-point measurements at different locations to scan the region of interest. Tomographic measurements of the inphase resistivity are usually referred to as electrical resistivity (or resistance) tomography (ERT). If polarization parameters are also imaged, then the method is called complex resistivity imaging (CRI) or electrical impedance tomography (EIT). The spectral extension is correspondingly referred to as sEIT 
(the terminology is not fixed; sometimes the ' $\mathrm{s}$ ' is omitted).

The resistivity in the subsurface is primarily determined by electrolytic conduction (water content and salinity) as well as surface conductivity (clay content, surface characteristics of biological matter) (e.g., Everett 2013). In the frequency range below a few $\mathrm{kHz}$, it can be assumed that the majority of lowfrequency polarization effects measured using sEIT is caused (directly or indirectly) by so-called electrical double layers (EDLs), that is regions with strong ion concentration gradients that form at charged surfaces such as clay particles or biomembranes, or constricted pores or cell clusters (e.g., Lyklema et al. 1983; Lyklema 2005). The resulting electromigrative relaxation processes exhibit a dominant relaxation time that can be related to certain physical parameters in soil, such as dominant pore or grain size or hydraulic conductivity (e.g., Schwarz 1962; Kemna et al. 2012; Revil et al. 2012, 2013). In biomatter the EDL characteristics are influenced by ion fluxes and the general physiological state of the system. In earlier work we hypothesized that spectral electrical polarization measurements can be correlated to structural and physiological properties of root systems (Weigand and Kemna 2017).

Resistivity methods, both on single electrode spreads and in imaging frameworks, have been used to detect water dynamics in the rhizosphere in multiple studies on tree root systems (e.g., Amato et al. 2008, 2009), in maize fields (Srayeddin and Doussan 2009), and in wheat fields (Whalley et al. 2017). Werban et al. (2008) imaged diurnal water dynamics in the rhizosphere of a pot experiment. Recently, Mary et al. (2018) have proposed a variant of resistivity tomography, with current injection into the stem of vine plants, in order to gain information on the distribution of fine root hairs, finding a good correlation to expected rooting depths.

Electrical polarization measurements have been conducted on biomatter for several decades now (e.g., Schwan 1957). Capacitance measurements focusing on root systems were first reported by Chloupek (1972) and Chloupek (1976), and multiple studies have been conducted since (for recent overviews, see Heřmanská et al. 2015; Cseresnyés et al. 2016). Also, a few studies measured multi-frequency capacitance or polarization signatures (e.g., Ozier-Lafontaine and Bajazet 2005; Repo et al. 2014). Imaging applications using polarization signatures are still rare. Newill et al. (2014) imaged the water distribution in the root zone of rhizotron containers using capacitive measurement techniques. Recently, Liao et al. (2015) used 3D electrical capacitance tomography to recover the root body of plant systems in water, and Weigand and Kemna (2017) and Weigand (2017) adapted sEIT to the use on crop root systems in aqueous solutions and recovered $2 \mathrm{D}$ information on oilseed root systems in rhizotron containers at the laboratory scale. Such imaging of electrical polarization properties has so far seen only little attention, despite huge potential for root research.

In this study we build upon recent work (Weigand and Kemna 2017) and further investigate the possibilities of sEIT and EIS for crop root applications by presenting three laboratory experiments using oilseed plants. Our objective is to show that electrical properties, captured using sEIT and EIS, are sensitive to physiological processes. In the first experiment, a root system with a pronounced tap root is imaged using sEIT as a reference case. The second experiment comprises the continuous and simultaneous monitoring of two root systems with sEIT in a nutrient deprived environment. Here, one plant is decapitated at the beginning, while the other is left intact, implying different physiological states for the two root systems but identical ambient conditions. In the third experiment, an oilseed plant in nutrient solution is monitored using EIS in a high-speed time-lapse mode. Changes in the electrical signatures are monitored over the diurnal cycle, i.e., subject to alternating light and dark conditions, over multiple days.

\section{Material and methods}

Conducting sEIT measurements is a multi-step process (Where EIS measurements form the basis of the tomographic analysis). In this regard they differ in application complexity and ease-of-use from other common approaches that use only one electrode spread for measurements (e.g., Chloupek 1972; Ellis et al. 2013; Postic and Doussan 2016; Cseresnyés et al. 2016). In this section, the basic principles of measuring electrical properties are presented first, followed by our current understanding of electrical polarization processes in the root-soil continuum. Afterwards we discuss the steps required to compute electrical tomograms of the samples. 
Measuring electrical properties

Electrical measurements are based on four-electrode spreads, which use two electrodes to inject a current $\hat{I}$, while the potential difference (voltage $\hat{V}$ ) is measured between the remaining pair of electrodes. In the case of polarizing materials a complex, frequencydependent electrical impedance $\hat{Z}(\omega)$ results:

$\hat{Z}(\omega)=\frac{\hat{U}(\omega)}{\hat{I}(\omega)}=Z^{\prime}(\omega)+j Z^{\prime \prime}(\omega)$,

with $Z^{\prime}$ and $Z^{\prime \prime}$ being the real and imaginary parts of $\hat{Z}$, respectively, $\omega$ the angular frequency, and $j$ the imaginary number. The indication of the dependence on $\omega$ is usually dropped in favor of a more compact notation. The inverse of the impedance is the admittance $\hat{Y}=1 / \hat{Z}$.

The complex conductivity $\hat{\sigma}$, or its inverse complex resistivity, can be computed for homogeneous materials by incorporating the geometrical arrangement of all four electrodes in the so-called geometric factor $K$ :

$\hat{\sigma}=\frac{1}{K \hat{Z}}=\sigma^{\prime}+j \sigma^{\prime \prime}=|\hat{\sigma}| e^{j \phi}$,

with $\sigma^{\prime}$ and $\sigma^{\prime \prime}$ the real and imaginary parts of $\hat{\sigma}$, respectively. The (real-valued) geometric factor $K$ is a direct measure of the expected signal-to-noise ratio of a given four-electrode measurement, and is commonly used as a criterion to exclude data from tomographic analysis (e.g., Stummer et al. 2004). In Eq. 2, $\sigma^{\prime}$ quantifies the conduction properties, while $\sigma^{\prime \prime}$ quantifies the polarization properties. The magnitude of the conductivity is denoted by $|\hat{\sigma}|$, and $\phi$ is the phase shift between injected current and measured voltage. When measurements are conducted on materials with inhomogeneous electrical properties, the conductivity (2) is referred to as the 'apparent conductivity', $\hat{\sigma}_{\mathrm{a}}$ (its inverse being the 'apparent resistivity'), because it does only capture a spatially integrated average value. Tomographic inversion algorithms must then be used to infer spatially resolved images of conductivity $|\hat{\sigma}|$ and $\phi$.

\section{Electrical impedance tomography}

Electrical impedance tomography comprises the reconstruction of $2 \mathrm{D}$ or $3 \mathrm{D}$ complex conductivity distributions of a given subsurface region or laboratory sample. This reconstruction is based on multiple, suitably located four-electrode measurements that cover the region of interest (the spatial information content of each measurement can be assessed by its sensitivity distribution). The forward response of a given complex conductivity distribution (model) is computed by solving Poisson's equation over this 2D/3D space, thereby predicting the measurement data. The inversion process then iteratively changes the conductivity model to minimize the difference between the predicted (forward response) and the measured data. Thus a repeated cycle of forward and inverse computation steps takes place. We use the inversion code CRTomo (Kemna 2000) to compute images of $|\sigma|$ and $\phi$ separately for multiple frequencies. In favor of a consistent display format we only show derived images of $\sigma^{\prime}$ and $\sigma^{\prime \prime}$. Details on the modeling and inversion algorithms can be found in Kemna (2000).

Data points are weighted in the inversion using individual data error estimates, which are described using a linear relationship between resistance error and measured resistance, consisting of an absolute error level and a relative error level (e.g., LaBrecque et al. 1996). Specifics regarding the application, and shortcomings, of this error model with respect to the present application to rhizotron measurements are discussed in Weigand and Kemna (2017).

\section{Polarization signatures of root systems}

Based on the literature both in the fields of nearsurface geophysics and electrical root properties, we propose to analyze spectral electrical complex conductivity signatures using the following hypotheses, based on the formulation of Weigand and Kemna (2017):

1. The magnitude of the low-frequency polarization response of roots is related to the overall surface area comprised by EDLs in the root-rhizosphere system, including the inner root structure. EDLs are known to form at cell walls and plasma membranes, and may also form at ion-selective structures larger than individual cells, such as Casparian strips (e.g., hypodermis and endodermis). Total internal surface area comprised by EDLs increases with root system volume.

2. The characteristic relaxation time of the lowfrequency polarization response of root elements 
provides information on the average length scale at which the polarization processes take place. While it is not clear to which extent a discrimination of specific polarization processes (e.g., cell membrane polarization and polarization of the hypodermis) is possible, changes in the relaxation time should indicate changes in the length scales of the polarizing structures.

3. EDLs in the inner root system are influenced by ions (nutrients) in the sap, EDLs at the outer root surface are influenced by ion concentrations in the external solution. Thus, physiological processes that influence the availability, usage, and translocation of ions directly influence the measured low-frequency polarization response.

4. sEIT is a suitable non-invasive method to image and monitor magnitude and characteristic relaxation time of the low-frequency polarization response of root systems.

The primary objective of this work is to prove that root physiological processes affect the root electrical properties in terms of polarization magnitude and relaxation time as imaged with sEIT (or measured as an effective response with EIS). By studying root systems for different experimental setups and physiological stimuli, this work thus addresses hypotheses 1 (in the experiments, root volume and thus internal EDL surface area vary across the sEIT image plane), 3 and 4 . Since we do not intend to elucidate the structural origin of the observed electrical responses, the results of this study do neither support nor disprove hypothesis 2 , rather we refer to it for interpretation purposes.

\section{Data selection (filtering)}

Outliers were removed from the impedance measurements ('raw data') prior to applying the inversion algorithm in experiments 1 and 2, following Weigand and Kemna (2017). Our choice of data filtering criteria described below reflects a conservative approach taken with regard to data quality and plausibility to ensure stable and consistent inversion results for different time steps and frequencies.

- For experiment 1, only impedance magnitudes (resistances) ranging from $0.05 \Omega$ to $25 \Omega$ were retained, as were measurements with impedance phase values greater than $-60 \mathrm{mrad}$, but less than
5 mrad (note that physically reasonable complex resistivity phase values are negative). These thresholds were selected based on histogram analyses of the raw data, which assumes a close proximity (grouping) of most measurement values because the sensitive areas of the individual measurements vary only slightly as configurations shift through the measurement schedule.

- For experiment 2, only impedance magnitudes ranging from $0.05 \Omega$ to $10 \Omega$ were retained. Moreover, apparent resistivities outside the range $10-50 \Omega \mathrm{m}$ were filtered and only impedance phase values less than $1 \mathrm{mrad}$ were kept. Due to spurious signals observed at the electrodes, all measurements containing electrodes from the lower horizontal electrode line (see Fig. 1d) were removed from the data. In addition, to ensure good signal-to-noise ratios, only measurements with a geometric factor less than $1000 \mathrm{~m}$ were kept. Finally, after the application of these filtering criteria, EIT spectra were ignored if they did not retain at least $90 \%$ of the data below $300 \mathrm{~Hz}$ (i.e., at least 19 frequency data points).

No filtering was applied to the data of experiment 3 , as here the raw data were analyzed and no tomographic inversion was performed.

Debye decomposition scheme

Each EIS spectrum contains a large body of information in the form of complex impedance values for multiple frequencies. In order to extract robust identifiers for interpretation, it is common to analyze the spectra using phenomenological models. We here use the Debye decomposition (DD) scheme to describe the characteristics of differently shaped polarization spectra in terms of summarizing, integral parameters (e.g., Nordsiek and Weller 2008; Weigand and Kemna 2016a). These parameters can then be used to describe systematic changes in the electrical response of root systems over time. For tomographic results the EIS signatures associated with individual image pixels are analyzed separately and then displayed using the pixel coordinates.

The DD describes the complex conductivity $\hat{\sigma}(\omega)$ by a superposition of elementary Debye relaxation terms:

$\hat{\sigma}(\omega)=\sigma_{\infty}\left(1-\sum_{k=1}^{N} \frac{m_{k}}{1+j \omega \tau_{k}}\right)$, 
with $\sigma_{\infty}$ the conductivity in the high-frequency limit and, $m_{k}$ the $k$-th chargeability corresponding to the $k$ th relaxation time $\tau_{k}$. The $N$ relaxation times $\tau_{k}$ are fixed in the fitting process and homogeneously distributed over the log time range spanned by the inverse relationships $\tau_{\min }=\frac{1}{2 \pi f_{\max }}$ and $\tau_{\max }=\frac{1}{2 \pi f_{\min }}$, with minimum and maximum measurement frequencies $f_{\min }$ and $f_{\max }$, extended by two orders of magnitude to each side (Weigand and Kemna 2016a).

The resulting relative weights $m_{k}\left(\tau_{k}\right)$ form the relaxation time distribution (RTD), from which the integral (summarizing) parameters can be derived (e.g., Nordsiek and Weller 2008; Weigand and Kemna 2016a):

- The total normalized chargeability $m_{\text {tot }}^{\mathrm{n}}$ is a measure of the overall polarization of the measured medium, corrected for the effect of conductivity magnitude (e.g., Tarasov and Titov 2013; Weigand and Kemna 2016a):

$$
m_{\mathrm{tot}}^{\mathrm{n}}=\sigma_{0} m_{\mathrm{tot}}, \quad m_{\mathrm{tot}}=\sum_{k=1}^{N} m_{k} .
$$

- The mean logarithmic relaxation time $\tau_{\text {mean }}$ represents a weighted mean of the RTD distribution:

$$
\tau_{\text {mean }}=\exp \left(\frac{\sum_{k=1}^{N} m_{k} \log \left(\tau_{k}\right)}{\sum_{k=1}^{N} m_{k}}\right) .
$$

It can be used to describe shifts of the polarization strength along the frequency axis.

The open-source implementation of Weigand and Kemna (2016a) is used for the DD analysis. The iterative inversion scheme balances data misfit and smoothing constraints by means of a regularization parameter $\lambda$. Data noise, along with inherent illconditioning of the inverse problem, requires a careful weighting of the smoothing constraint. Larger $\lambda$ values cause a stronger smoothing of the resulting RTD, at the (possible) expense of the data fit quality.

Uncertainties in the measurements

No data uncertainties are reported in this study because the estimation of uncertainties from non-linear inversion schemes, such as used in the electrical imaging and the Debye decomposition steps, is not yet established but a topic of active research in the geophysical community. Coupled with the low number of repetitions for each individual measurement of a tomographic data set, we abstain from introducing possibly unreliable uncertainty estimations. However, we are confident that measurement uncertainties, where not explicitly discussed, do not interfere with the interpretation of our data for the following reasons: The sEIT measurement system used for the electrical measurements was designed for high accuracy (Zimmermann et al. 2008; Zimmermann et al. 2012), and the temporal evolution of the measurements, discussed later, also shows consistency which does imply a sufficiently low level of data noise.

\section{Classifying pixels into root groups}

Pixels of the finite-element mesh used for the inversion are partitioned into two groups after the DD is applied to the imaging results. As no significant polarization response can be expected in the considered frequency range from the water in the rhizotron, all polarization signals can be attributed to the embedded root systems. Therefore, the maximum recovered polarization response in the lower half of the rhizotron, i.e., the region free of root elements, is used as a lower noise threshold to classify pixels into two groups. Correspondingly, pixels exhibiting a total polarization $\left(m_{\mathrm{tot}}^{\mathrm{n}}\right)$ response above this threshold are assigned to the 'root' group, while all other pixels are assigned to the 'water' group. In addition, relaxation times are only analyzed for pixels with a distinct polarization signature, i.e., with a clear shape, and therefore are only shown for the 'root' group.

\section{Experimental setup and procedures}

The setup follows the rhizotron setup described in Weigand and Kemna (2017): The rhizotron container has a width of $30 \mathrm{~cm}$, a height of $78 \mathrm{~cm}$, and a depth of $2 \mathrm{~cm}$, and is equipped with 38 brass electrodes that are fixed on the front plate of the rhizotron. To reduce electrode polarization effects that would be expected for brass material, the electrodes do not protrude into the internal volume (for a thorough investigation of this issue, see Zimmermann et al. 2012). This fully non-invasive setup comprises a pseudo-2D scenario in which the extension of the root system(s) can be 
visually verified through the transparent front plates. The sEIT/EIS measurements were conducted with the EIT40 system designed by Zimmermann et al. (2008).

Small inconsistencies arise between the measurements and the predicted response of the forward modeling code due to the imperfect 2D situation. These inconsistencies are accounted for by applying correction factors to the measured impedance data before the inversion. The correction factors are computed using separate measurements on the rhizotron filled with water of known conductivity. A detailed description of the computation and application of these correction factors is found in Weigand and Kemna (2017).

In the following, three laboratory experiments are presented and discussed. In all experiments root systems were embedded in water or nutrient solution. This ensured that electrical polarization could only originate in the root systems, thus allowing us to investigate these signals without interference from soil or substrates.

\section{Experiment 1: sEIT imaging of a single plant root system}

Basic sEIT imaging characteristics are presented using a single-time-step measurement on one oilseed plant (Brassica napus L.). The plant was taken from the
Klein-Altendorf experimental research station near Bonn, Germany (e.g., Gaiser et al. 2012), and after washing, was kept in hydroponics for a few days. Correspondingly, it exhibits much thicker root elements than produced by plants grown entirely in hydroponics. The root system was placed on the right side of a rhizotron container filled with tap water for the duration of the sEIT measurements (Fig. 1a). Measurements were conducted at 35 frequencies between $0.46 \mathrm{~Hz}$ and $45 \mathrm{kHz}$. Imaging (and subsequent DD) was conducted only on the frequency range between 0.46 and $300 \mathrm{~Hz}$ (22 frequencies) to exclude possible errors introduced by electromagnetic coupling effects for higher frequencies. After data processing (filtering), all frequencies retained at least 392 measurements (457 maximum). Measurement configurations primarily consisted of dipole-dipole skip-0 and skip-1 measurements, as well as 'cross-rhizotron' configurations, in which one current electrode is located on either side of the rhizotron, with potential electrodes located below or above these electrodes. For dipole-dipole configurations, the 'skip' refers to the distance (in electrodes) between electrodes of a given current/potential dipole (e.g., Telford et al. 1990). For example, a skip-1 dipole-dipole configuration skips 1 electrode between electrodes used for current/potential measurements.
Fig. 1 Experimental setups of the three presented experiments: a experiment 1 (imaging of root extension), b experiment 2 (time-lapse imaging during nutrient deprivation), c sketch of the finite-element domain used for the inversions in experiments 1 and 2, d experiment 3 (spectral timelapse monitoring over the diurnal cycle). Black dots denote electrode positions, with indicated numbering

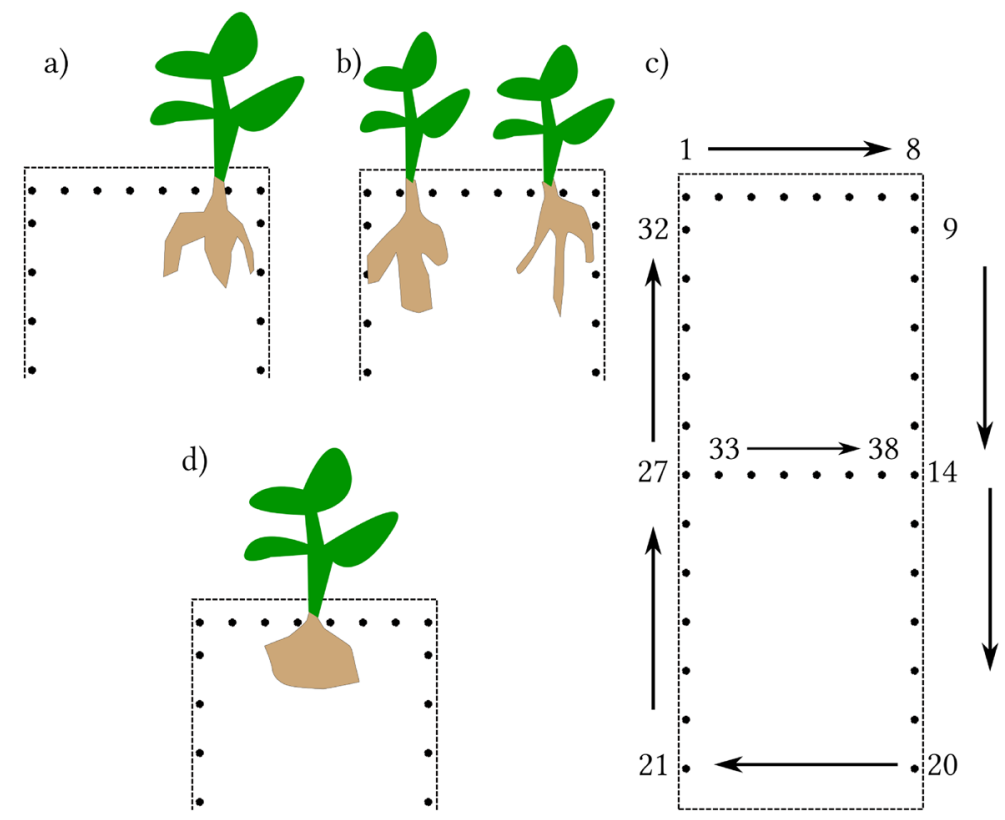


Experiment 2: sEIT monitoring of two root systems

The second experiment comprises the concurrent imaging of two field plants from the Klein-Altendorf research station. The plants were washed and kept in hydroponics before the experiment for several days. During the experiment they were placed next to each other in the rhizotron container (Fig. 1b), and the right plant was decapitated 44 hours before the first time step presented in this study, while the left plant was left intact. The rhizotron was filled with tap water, which induced a nutrient stress situation in the plants. Continuous EIT measurements were conducted over five days, with each data set recorded in approximately 4 hours. Light was turned on at 6:30 a.m. and turned off at 8 p.m. each day. Between measurement sets, the rhizotron container was ventilated to prevent the emergence of an anaerobic environment.

Between hours 55 and 75, both plants were removed from the rhizotron and placed in nutrient solution for 20 hours. Then, they were rinsed and reinserted into the rhizotron.

The same measurement configurations as in experiment 1 were used. Measurements were conducted at 35 frequencies between $0.46 \mathrm{~Hz}$ and $45 \mathrm{kHz}$. Imaging (and subsequent DD) was performed only on the frequency range between 0.46 and $300 \mathrm{~Hz}$ (22 frequencies), where larger errors due to electromagnetic coupling can be neglected.

The sEIT monitoring results presented in this study begin 48 hours after inserting the plants in the rhizotron. This was done for technical reasons (testing of the equipment during this time, and to accommodate for a tuning-in phase of the plant to the new environment).

\section{Experiment 3: EIS monitoring with high temporal resolution}

In the third experiment, one oilseed plant (completely grown in hydroponics) was placed in the rhizotron filled with nutrient solution (Fig. 1c). Day/night cycles were simulated by operating an LED growth lamp in 12 hour intervals from 7 a.m. to 7 p.m., thus creating a stable environment for the plant. Continuous EIS measurements on one four-electrode spread, located directly around the stem, were then conducted over 30 days with an individual measurement time of approx. 7 minutes. At two times during the experiment, the growth lamp was not turned on during daylight hours, thus creating periods of prolonged darkness. Measurements were conducted at 46 frequencies between $0.1 \mathrm{~Hz}$ and $45 \mathrm{kHz}$, with 29 frequencies below 320 $\mathrm{Hz}$ being used for the subsequent DD analysis of the impedance spectra.

\section{Results}

In this section results for all three experiments are presented and discussed, followed by an overall discussion of the experiments.

Imaging the spatial extension of root systems in water

This sEIT measurement serves to investigate the imaging capabilities of the system and to analyze the spectral characteristics that can be recovered from the images. Only a weak anomaly of the in-phase conductivity (Fig. 2b) indicates the spatial extension of the root system (Fig. 2a), located on the right side of the rhizotron. However, polarization parameters $\left(\sigma^{\prime \prime}\right)$ recovered from the inversion results show a significant polarization anomaly in the region of the root system (Fig. 2c). Comparison of the $\sigma^{\prime \prime}$ image to a photograph of the setup (Fig. 2a) shows the resolution limits of the method for the given setup, which does not allow the imaging of individual root elements. Rather the area with roots present is delineated. (The spatial resolution of sEIT is directly related to the spacing of the electrodes.)

Spectral polarization signatures extracted from the imaging results at certain locations (colored dots in Figs. 2b,c) show a general decrease in signal strength with increasing distance from the central part of the root system (Fig. 2d and supplement Fig. S1). However, the spectra retain their typical shape in the vicinity of the root system. The observed decrease in polarization strength for the different locations is stronger for lower frequencies $(\leq 10 \mathrm{~Hz})$, indicating a stronger spatial signal variation in this frequency range. Far away from the root system (red dot) a negligible polarization response is found, roughly two orders of magnitude smaller compared to the region with root elements (blue dot).

The spectral behavior of the signatures is also reflected in the imaging results of the Debye decomposition, which takes into account all frequencies 


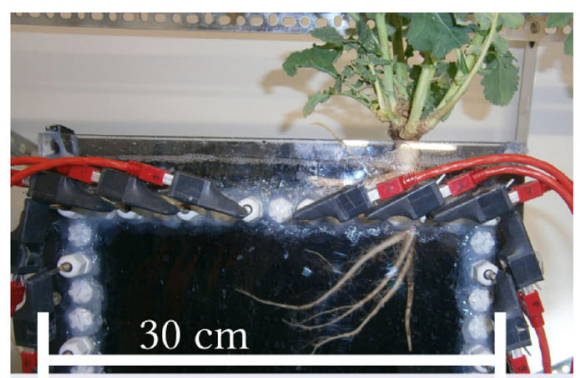

c)

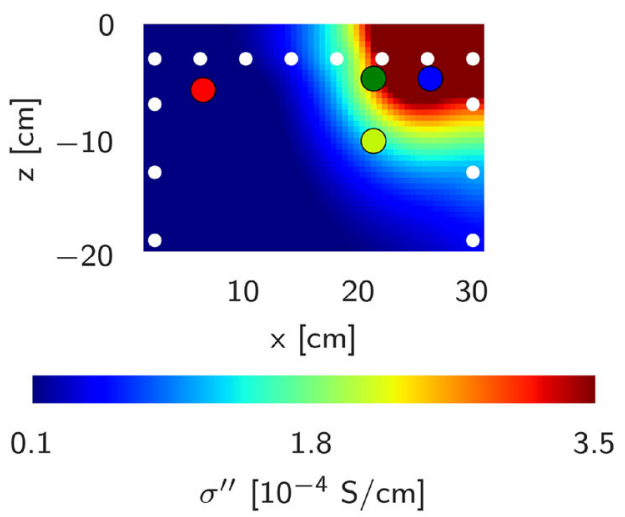

d) b)

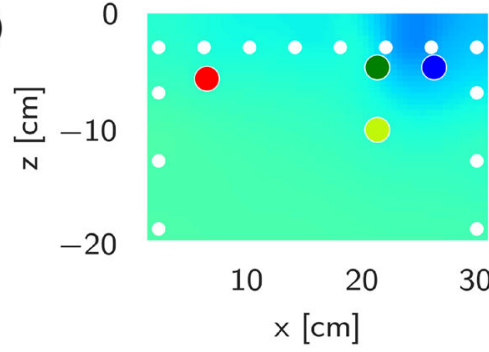

0.03

$\sigma^{\prime}[\mathrm{S} / \mathrm{m}]$

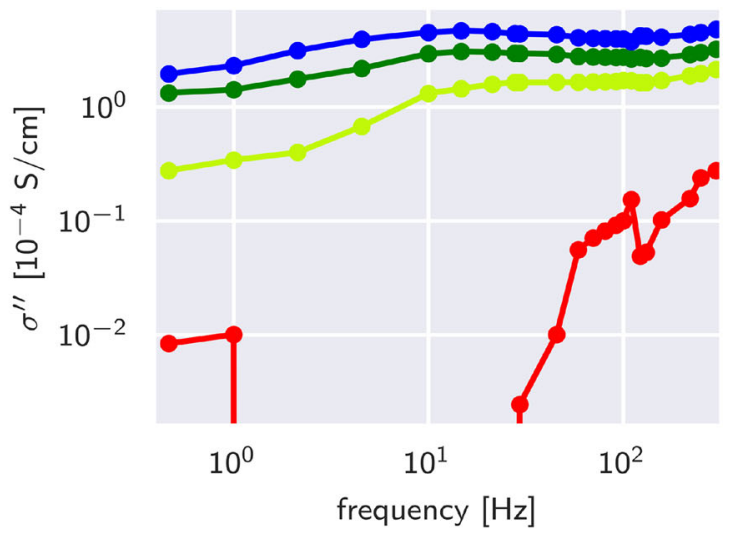

Fig. 2 a Photograph of experiment 1. b, c Single-frequency imaging results for $\sigma^{\prime}$ (b) and $\sigma^{\prime \prime}$ (c) at $10 \mathrm{~Hz}$. The colored dots indicate positions from which spectral signatures (intrinsic spectra) are extracted and plotted in (d)

up to $300 \mathrm{~Hz}$ (Fig. 3, exemplary $\sigma^{\prime \prime}$ spectra used for the decomposition are shown in Fig. 2d). Total chargeabilities $m_{\text {tot }}^{\text {n }}$ (Fig. 3a) exhibit a pattern consistent with the $\sigma^{\prime \prime}$ imaging result at $10 \mathrm{~Hz}$ (Fig. 2c). Relaxation times, recovered for regions with sufficient polarization strength (see section 'Classifying pixels into root groups'), show systematically increasing values towards the stem region of the root system (c.f. Figs. 2a and 3b).

The results presented in Figs. 2 and 3 confirm those reported in earlier work (Weigand and Kemna 2017), both in terms of polarization $\left(m_{\mathrm{tot}}^{\mathrm{n}}\right)$ strength

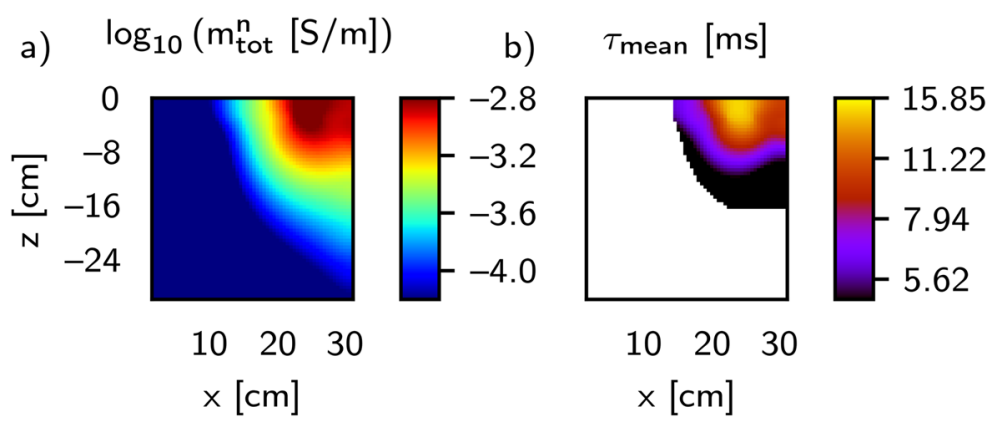

Fig. 3 Experiment 1: Images of spectral parameters recovered from the per-pixel analysis of intrinsic signatures using the Debye decomposition scheme. a total chargeability $m_{\mathrm{tot}}^{\mathrm{n}}, \mathbf{b}$ mean relaxation time $\tau_{\text {mean }}$. Plotted are only pixels with an $\log _{10}$

$m_{\text {tot }}^{\mathrm{n}}$ value above -3.8 corresponding to the largest polarization response in the lower half of the rhizotron, where no polarizable material is located 
and relaxation times. However, the root system in this experiment has much thicker root elements compared to the root systems used in Weigand and Kemna (2017), which were completely grown in hydroponics. The measurement of similar polarization strengths (in terms of $m_{\mathrm{tot}}^{\mathrm{n}}$ ) supports hypothesis no. 1 (see section 'Polarization signatures of root systems'), which states that the overall polarization magnitude is controlled by the total surface area comprised by EDLs (rather than by root diameter). Such surface area may be provided by many thin roots or a few thick roots. Slightly surprising is the fact that the relaxation time values of this experiment more or less match those reported by Weigand and Kemna (2017) for the beginning of their experiment, despite both experiments using differently structured root systems. According to hypothesis no. 2 we expect the relaxation times to provide information on the length scale at which the polarization processes take place. Similar relaxation times recovered for these two different root systems suggest that this length scale is not controlled by root diameter. Two aspects should be kept in mind when interpreting, and especially comparing, the presented results: First, the measurement setup changed between this experiment and the one described in Weigand and Kemna (2017) in terms of measurement configuration and location of the root systems. This influences the spatial resolution of the electrical measurements, but should not strongly influence the recovered relaxation time values (Weigand et al. 2017). Second, no detailed biochemical analysis was conducted in the experiments; $\mathrm{pH}$, nutrient content, and other environmental conditions were not recorded.

Monitoring of root systems under nutrient deprivation

The second experiment comprises the simultaneous measurement of two root systems in one rhizotron container. Such a setup avoids possible problems associated with the comparison of different experiments in terms of electrical responses by ensuring consistent environmental conditions for both test plants. Two different stimuli were applied to the plants in this experiment: First, both root systems were submerged in tap water to create a nutrient-deprived environment. Second, the right plant was decapitated shortly before the experiment, which allowed a direct comparison of the electrical response of both root systems to the nutrient deprivation.
The spatial resolution capabilities are analyzed using the tomographic results for $\sigma^{\prime}$ and $\sigma^{\prime \prime}$ for the first time step at $10 \mathrm{~Hz}$ (Fig. 4b and c). Similar to the results shown for experiment 1 , the ohmic signatures do not show significant anomalies that can be attributed to the root systems (Fig. $4 a$ and b). Also in line with the previous experiment, clear polarization anomalies can be identified at the locations of both root systems (Fig. 4a and c). However, the anomalies show decreased signal strengths for elements of the main root system that are located further away from the electrodes in the center of the rhizotron (Fig. 4c). In addition, areas coinciding with the root systems also exhibit clear spectral signatures (Fig. 4d, red (1), pink (2), and green (3) curves). As previously observed, areas with no roots do not show significant polarization (grey (4) and dark-blue (5) curves).

The conductivity distribution, as recovered from the DD of complex conductivity spectra (exemplary $\sigma^{\prime \prime}$ spectra are shown in Fig. 4d and Figure S2), shows only slight changes over time (Fig. 5). At hours 91 and 112 high-conductive anomalies emerge at the top right corner of the rhizotron. Due to their sporadic occurrence we attribute these anomalies to inversion artifacts.

Images of the total polarization (in terms of $m_{\text {tot }}^{\mathrm{n}}$ ) show a steady decrease in signal strength over time for the left, intact plant (Fig. 6, left panel). Contrary to this, the decapitated plant only shows minor variations in the observed patterns and signal strength. We attribute these to small variations in the analysis procedures (i.e., influence of the inversion process). While no clear changes can be detected between images before and after the plants were placed in the nutrient solution (between hours 44 and 75, indicated by the asterisk in the figure), the temporal evolution of the mean $m_{\text {tot }}^{\text {n }}$ value clearly indicates that the overall decrease in the polarization signal for the left plant stopped when it was placed in nutrient solution (Fig. 7, blue curve). Again, the decapitated plant shows only minor variations in the polarization strength (green curve).

The relaxation times show a behavior similar to $m_{\mathrm{tot}}^{\mathrm{n}}$ : The intact plant shows a small, but consistent, decrease in relaxation times (Fig. 8, left side), while the decapitated plant does not show significant changes (Fig. 8, right side). Note that some artifacts are present for $\tau_{\text {mean }}$ at the edges of the root zones. We attribute these to the pixel filter, which categorizes 

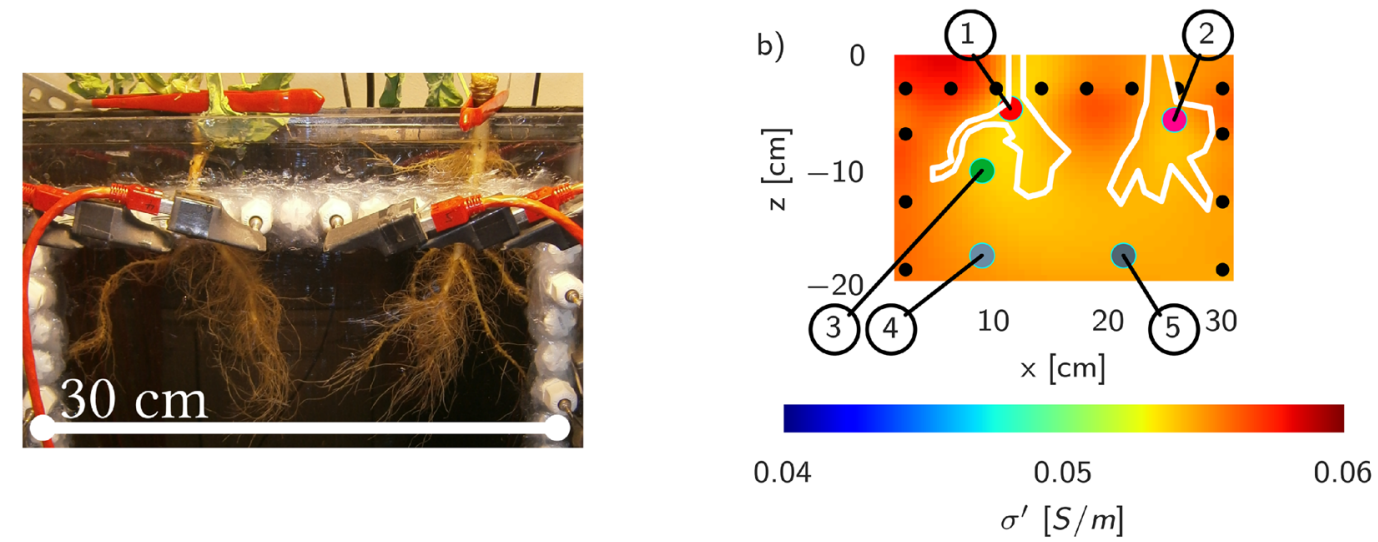

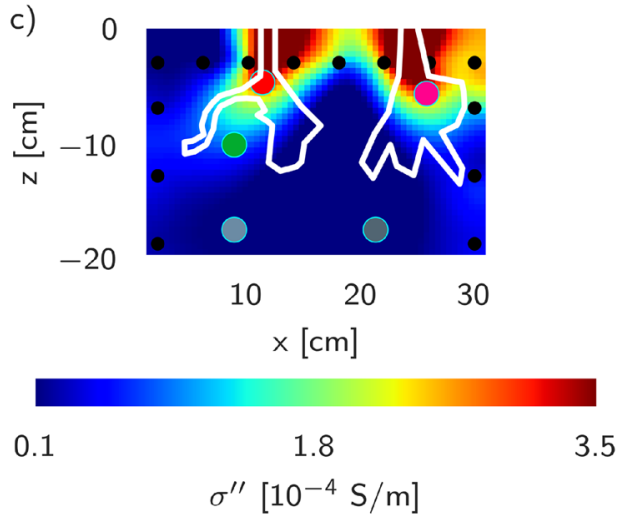

Fig. 4 a Photograph of the root systems. b, c Single-frequency inversion results of first time step for both root systems of experiment 2 at $10 \mathrm{~Hz}$. b) $\sigma^{\prime}$ image, c) $\sigma^{\prime \prime}$ image. The approximate extension of the root system, recovered from photographs, is

pixels either as belonging to the root zone or to the water region. As the boundary between root zone and free water is fluid and heavily influenced by the smoothing imposed by the inversion, no weight should be given to the interpretation of these regions (Fig. 8).

It is well known that plants can regulate water flux and nutrient transport in response to nutrient availability (e.g., Clarkson et al. 2000; Cabañero and Carvajal 2007; Cramer et al. 2009). Also, there are complex signaling schemes at work that communicate nutrient stress from the roots to the shoot, thereby allowing the whole plant system to react accordingly (e.g., Clarkson et al. 2000; Cramer et al. 2009), e.g., by decreasing root hydraulic conductance and translocating nutrients to the shoot or the root. It has also been shown that plants can, to some extent, store and reuse certain nutrients during stress periods (e.g., shown for $\mathrm{NO}_{3}^{-}$ usage by van der Leij et al. 1998). d)

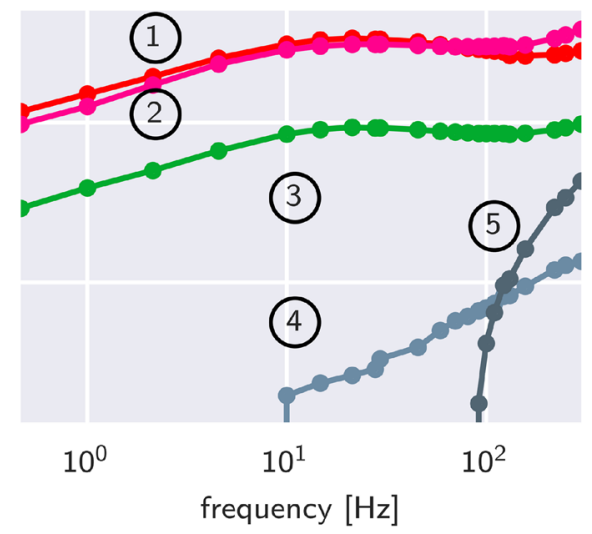

indicated by the white contours. d) Intrinsic spectra $\left(\sigma^{\prime \prime}\right)$ recovered at different positions of the imaging results. Positions are marked in Figs. a,b by color-coded dots and by numbers in Figs. b,d

Ionic composition next to, and ion fluxes across, plant cell membranes are interlinked with the transmembrane electric potential difference as well as the electric surface potential (and correspondingly electric surface charge) at the membrane (e.g., Kinraide et al. 1998; Kinraide and Wang 2010), which are in turn controlling the strength of the EDL forming at the membrane. Therefore the physiological responses described above suggest that nutrient deprivation will directly affect such EDLs and thus the electrical polarization response of the root system.

Following these principles, we interpret this experiment as follows: The intact plant system (left side of the figures) had been subject to a prolonged nutrient deprivation, eventually decreased its physiological activity and died off. Due to its structural integrity long-distance ion transport from the roots to the leaves was still possible, although possibly mitigated by the 


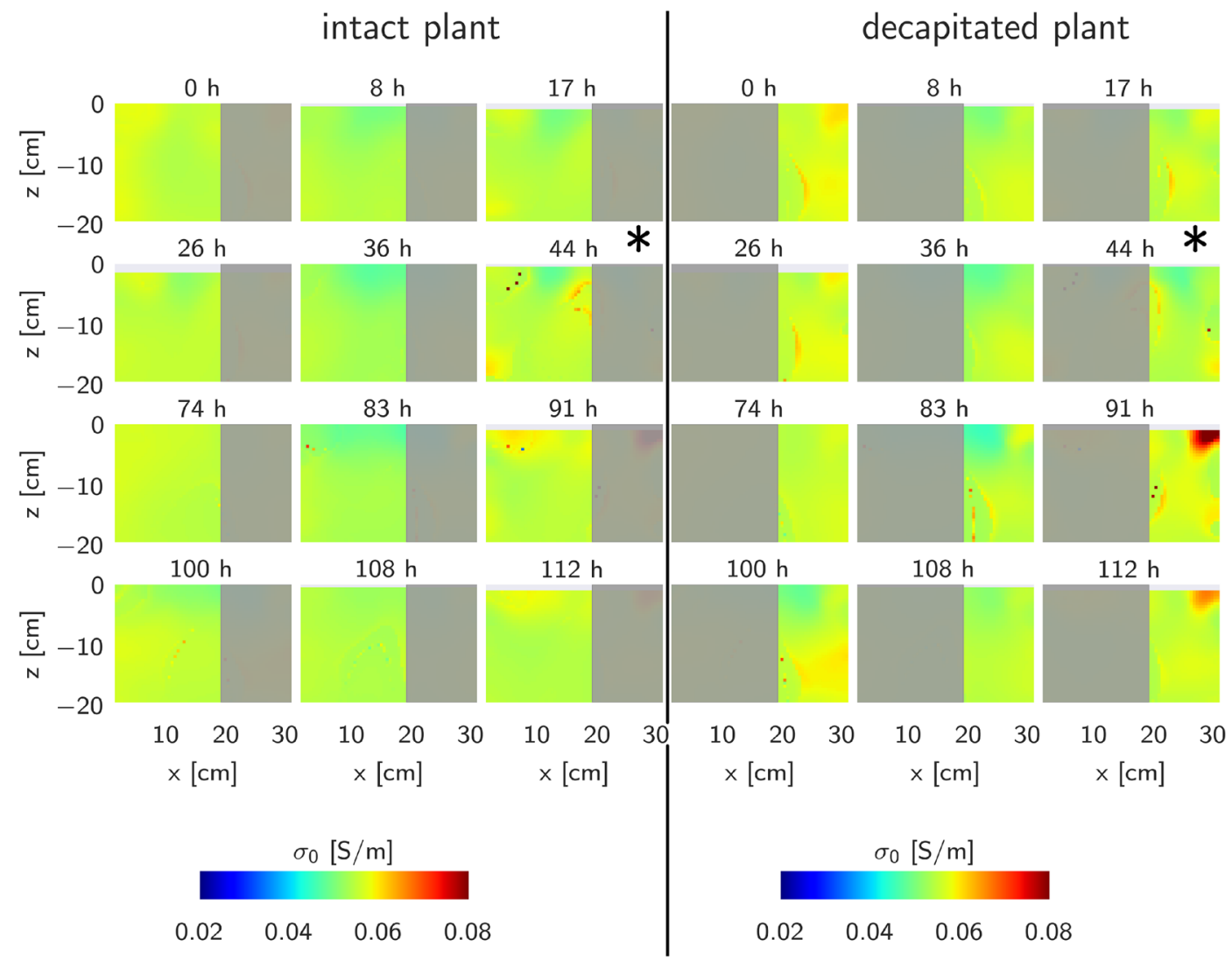

Fig. 5 Images of the Debye decomposition parameter $\sigma_{0}$ for both plants at various time steps. The images are split into two, with gray areas indicating the part shown on the other side of the figure. Left part: intact plant, right part: decapitated plant. Only

aforementioned physiological reactions. The observed reduction of electrical polarization strength (Fig. 6, left panel) suggests that the continuous redistribution and consumption of nutrients went along with a weakening of the EDLs in the root system.

The decapitated plant (right side of the figures), however, has lost its ability to transport nutrients over longer distances to and from the shoots, due to the destroyed hydraulic transport mechanisms. Thus, only osmotic and diffusive transport mechanisms remained, which do not sustain effective long-distance transport. In addition, physiological activity in the decapitated root system was strongly reduced, and signaling pathways that are normally used in response to stress were not available anymore due to the critical shoot components missing. Correspondingly, demand for nutrients was strongly suppressed. The observed preservation of the electrical polarization response (Fig. 6, right the upper half of the rhizotron is shown. The asterisk indicates the last measurement before the plants were placed in nutrient solution for 20 hours

panel) suggests that the root system entered a steady state, both physiologically and electrically, until it began to structurally decompose at some point.

Clarkson et al. (2000) also showed that a re-supply of $\mathrm{NO}_{3}^{-}$can restore the hydraulic conductance of roots. In this light the stabilization of polarization strength observed for the intact plant after the root systems were temporarily placed in nutrient solution could be interpreted as a manifestation of associated stabilization processes within the root system (Fig. 7). This observed behavior is consistent with the experiment previously reported in Weigand and Kemna (2017). The decapitated plant, on the other hand, is not expected to show significant nutrient uptake in this situation due to the missing long-distance nutrient transport capability.

From a methodological point of view this experiment highlights some of the challenges involved in 


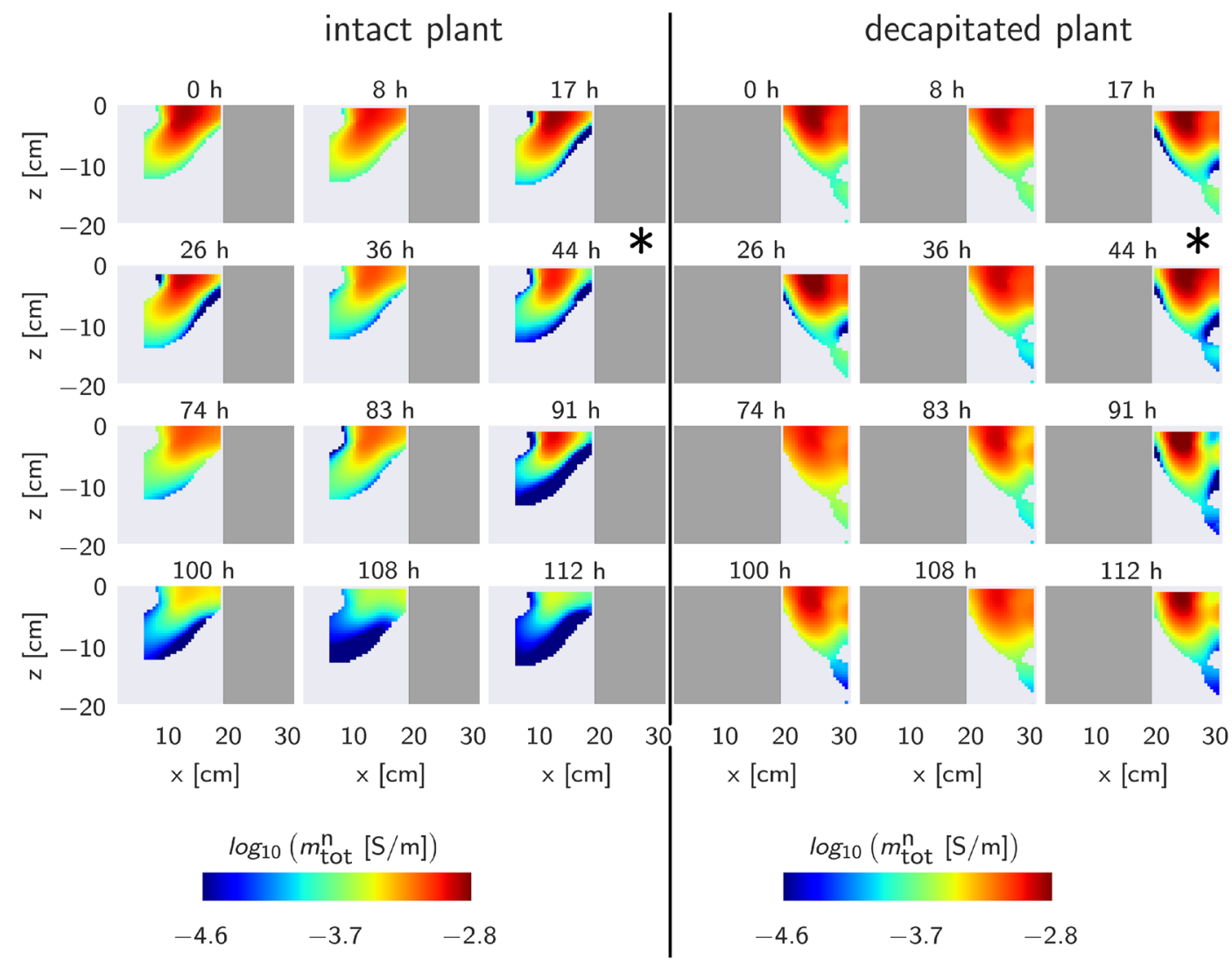

Fig. 6 Selected images of the Debye decomposition parameter $m_{\text {tot }}^{\mathrm{n}}$ for both plants at various time steps. The images are split into two, with gray areas indicating the part shown on the other side of the figure. Left side: intact plant, right side:

the simultaneous measurement of two plants in one rhizotron: The recovered spatial extents of the root systems are not as accurate as those recovered in experiments with only one root system in the rhizotron (e.g., Fig. 5 or Weigand and Kemna 2017). This can be attributed to the limits of spatial resolution imposed by the number and location of electrodes, the used measurement configurations, and by the method itself. We note, however, that with the present setup consistent information (especially by analyzing the frequency dependence of the electrical properties) is recovered on the root system extension and its temporal evolution in the course of the experiment.

\section{Monitoring of a root system over multiple diurnal} cycles

The third experiment presented in this study focuses on the measurement of spectral signatures with high decapitated plant. Shaded areas denote masked rhizotron sides. Only the upper half of the rhizotron is shown. The asterisk indicates the last measurement before the plants were placed in nutrient solution for 20 hours

temporal resolution. No tomographic data were recorded in favor of fast data acquisition. Only a single four-electrode configuration, located directly above the root system, was used to capture daily variations in

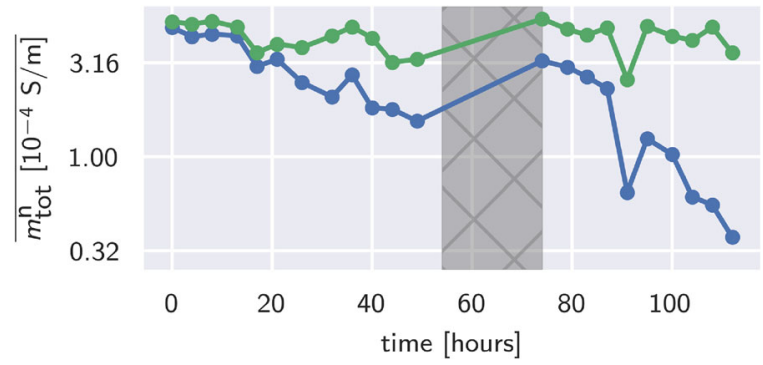

Fig. 7 Time evolution of mean $m_{\text {tot }}^{\mathrm{n}}$ values for the left (blue) and right (green) root system regions. The time spent in nutrient solution is indicated by the shaded region. Only pixels associated with the presence of root elements were used to compute the average values 


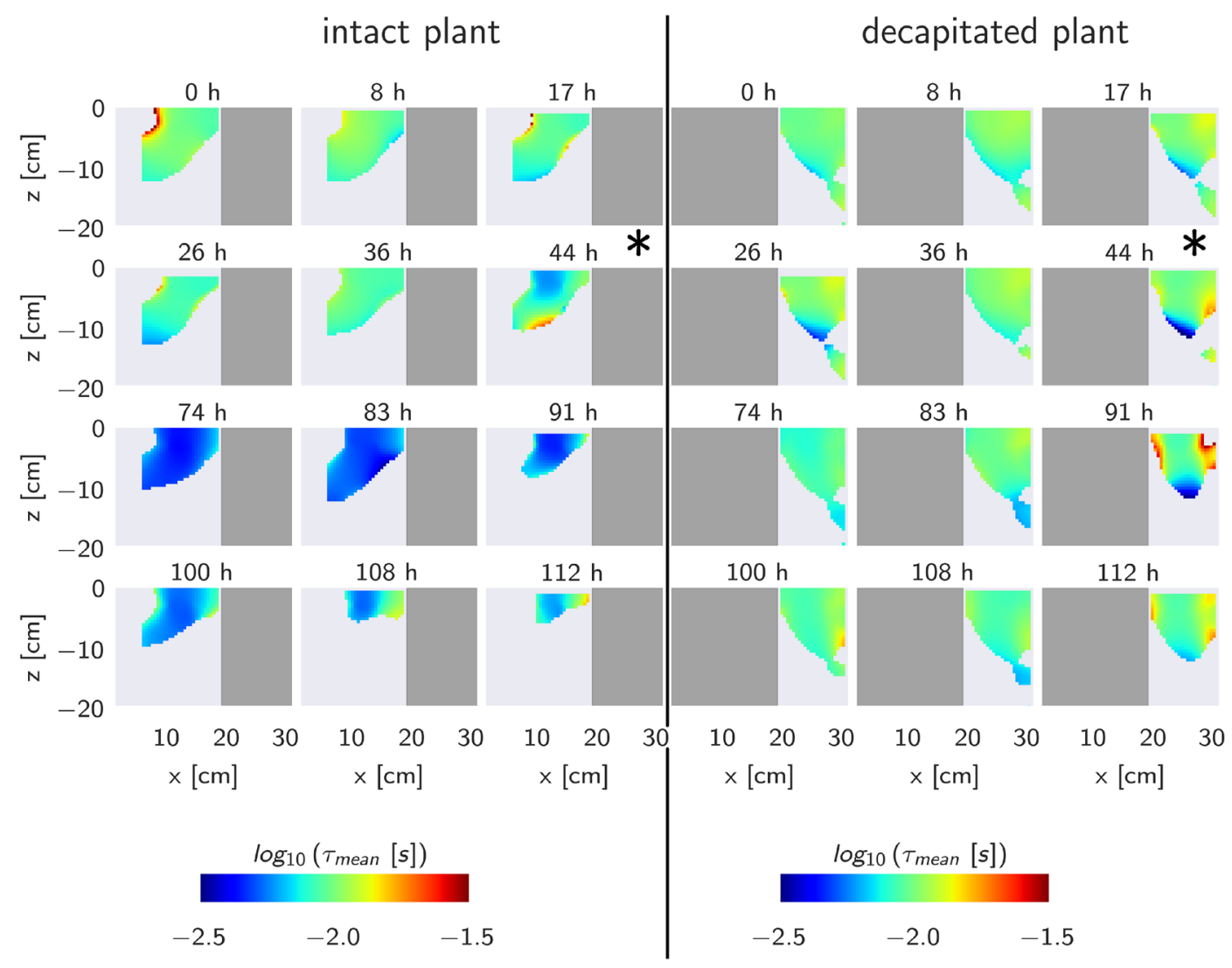

Fig. 8 Selected images of the Debye decomposition parameter $\tau_{\text {mean }}$ for both plants at various time steps. The images are split into two, with gray areas indicating the part shown on the other side of the figure. Left side: intact plant, right side: decapitated

the electrical spectral signatures of an oilseed canola root system embedded in nutrient solution.

\section{Background measurements without roots}

Reference measurements were conducted on a rhizotron without an embedded root system. Measured $\sigma_{0}$ values show only marginal variations (Fig. 9a), and polarization responses show no systematic patterns between dark and light periods (Fig. 9b).

\section{Monitoring $\sigma_{0}^{\mathrm{a}}$ on the root system}

The measured apparent conductivity varies on a daily basis, with maxima during daylight hours and minima during dark conditions (Fig. 10a and b). Exceptions in the diurnal dynamics occur on two occasions when the growth lamp was left off during daylight hours, plant. Only the upper half of the rhizotron is shown. The asterisk indicates the last measurement before the plants were placed in nutrient solution for 20 hours

thus creating prolonged darkness periods (Fig. 10c and d). Here, conductivity stayed at low values and varied to a much smaller degree, compared to the regular diurnal cycles. Daily extrema varied between subsequent days, but on smaller scales than the observed dynamics between dark and light periods (ca. 15 - 30 $\%$ change over the 24 hour cycle, depending on the individual peaks).

As both measurements, with and without root system, were made under similar conditions, temperature effects are highly unlikely as a possible cause for the observed diurnal changes. Ambient air temperature changes during the experiment were below 2 degree Celsius. Depending on specifics such as ion concentration, electrolytic conductivity typically increases between 2 and $4 \%$ per degree Celsius (e.g., Sen and Goode 1992). Therefore temperature effects cannot explain the observed temporal variations $(15-30 \%$ for 
Fig. 9 Spectral parameters recovered from the DD of apparent complex conductivity measurements on a rhizotron solely filled with nutrient solution (no root system present). a Low-frequency in-phase conductivity $\sigma_{0}^{\mathrm{a}}$, b total normalized chargeability $m_{\text {tot }}^{\text {n,a }}$

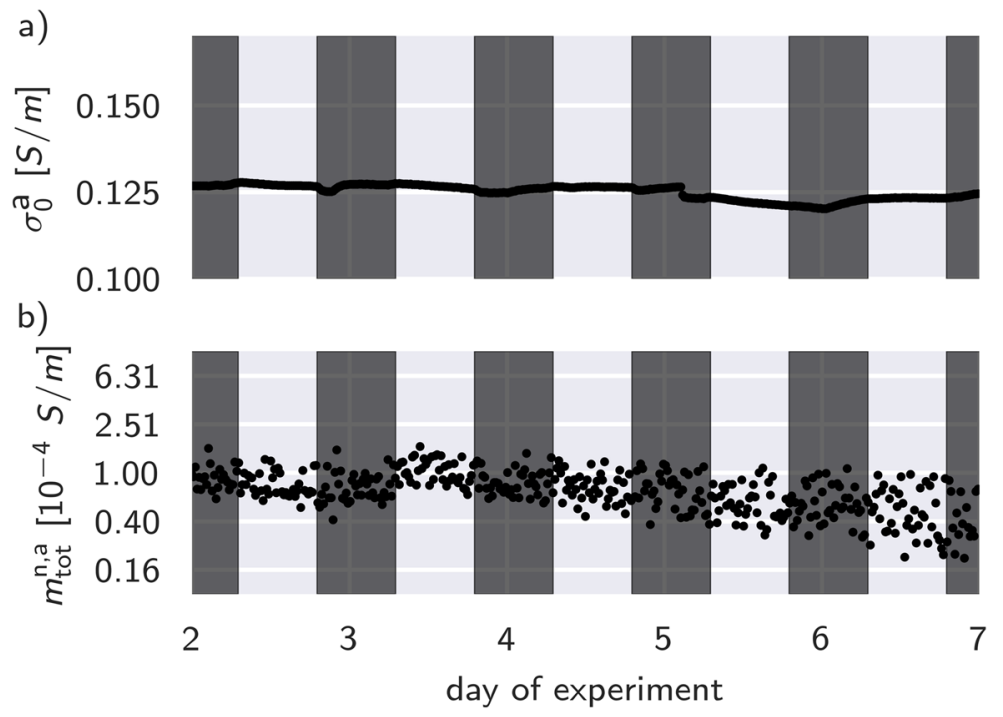

at the end of the darkness periods, with a subsequent relaxation towards a minimum during daylight hours. On some days (e.g., days 10 and 17) $m_{\text {tot }}^{\text {n, a }}$ peaks slightly before the light is turned on again. During prolonged darkness, polarization strength generally increases over the whole period of darkness, although smaller peaks are observed at the natural daylight intervals (Fig. 11c,d). It should be noted that the polarization peaks are shifted with respect to the dark/night switch-over times, and are shifted with respect to

a)

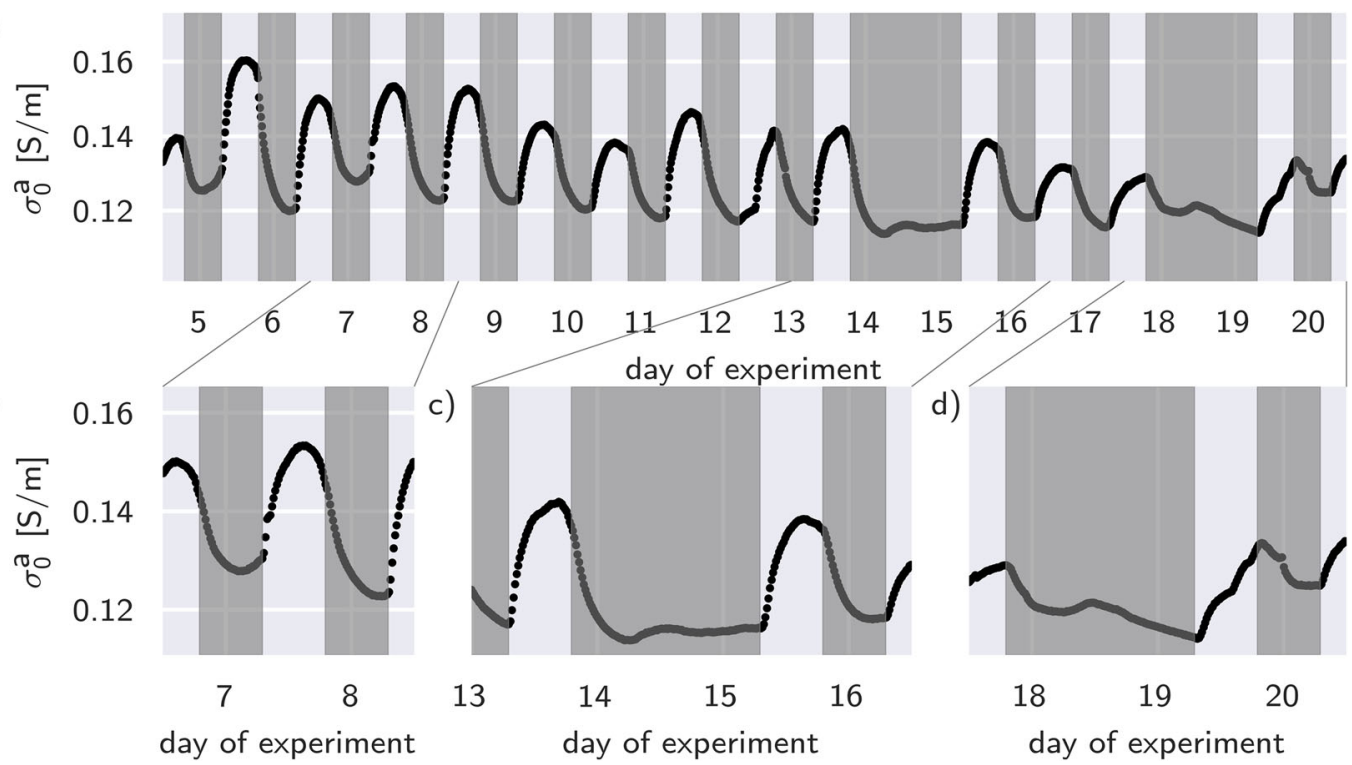

Fig. 10 Time series of the parameter $\sigma_{0}^{\mathrm{a}}$, as recovered from the DD of the EIS measurements on the root system. Shaded regions indicate time spans in dark conditions, i.e., with the growth light turned off. Selected time spans are enlarged in the lower row (b-d) 


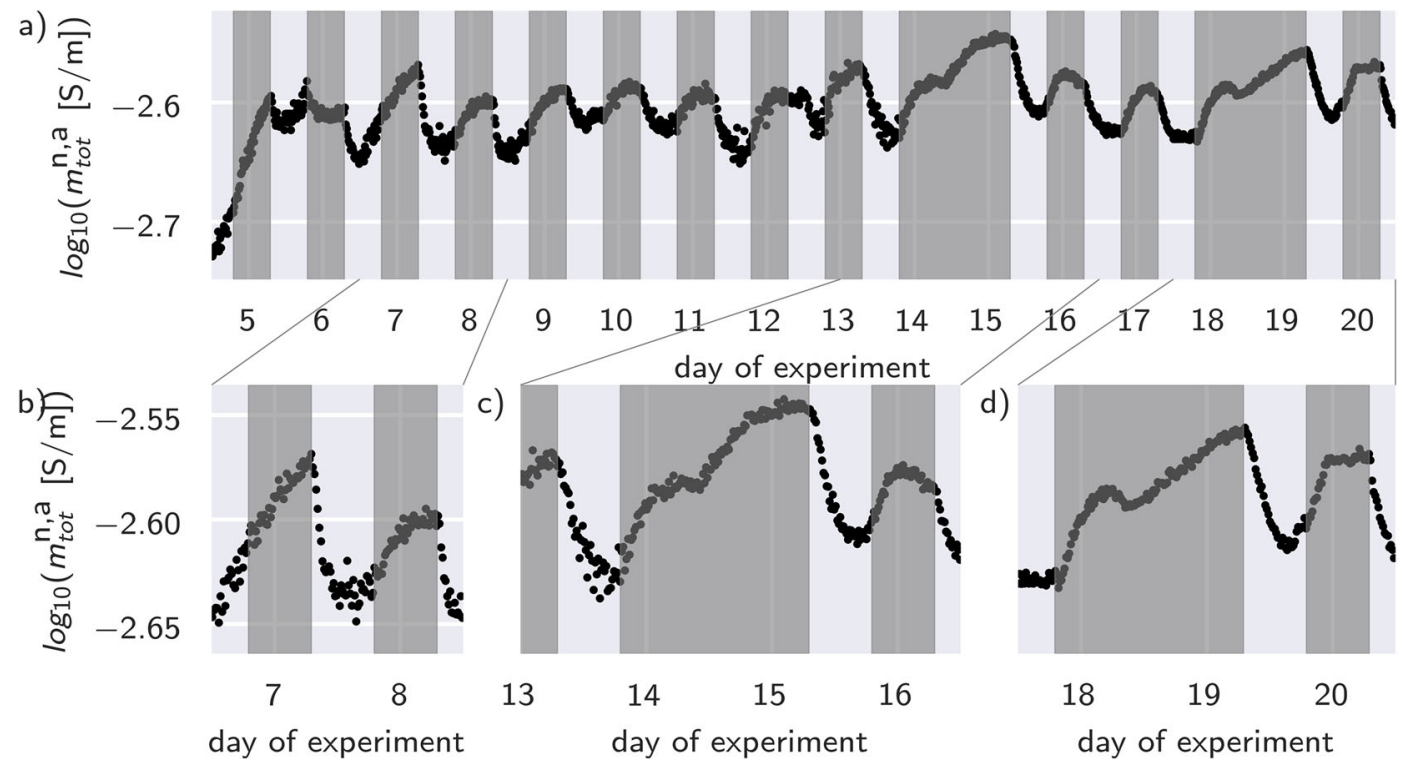

Fig. 11 Time series of the parameter $m_{\text {tot }}^{\mathrm{n}, \mathrm{a}}$, as recovered from the DD of the EIS measurements on the root system. Shaded regions indicate time spans in dark conditions, i.e., with the

$\sigma_{0}^{\mathrm{a}}$ and $\tau_{\text {mean }}$ (see also supplement Figure $\mathrm{S} 4$ for an overlay of all three parameters for a selected time span).

Polarization magnitudes are nearly two orders of magnitude larger than for the background measurements (Fig. 9b). Also, the background measurements do not exhibit clear diurnal cycles. Therefore, as with $\sigma_{0}^{\mathrm{a}}$, we rule out temperature effects for the observed variations in $m_{\mathrm{tot}}^{\mathrm{n}, \mathrm{a}}$, too.

\section{Relaxation time}

Mean relaxation time $\tau_{\text {mean }}$, recovered from the DD, does not show clear patterns that correlate with the diurnal cycles, although a certain trend towards increasing relaxation times during dark hours can be detected using a moving average filter (Fig. 12b and c). However, during the periods of prolonged darkness, $\tau_{\text {mean }}$ shows a clear increase towards larger relaxation times (Fig. 12c and d). This trend reverses as soon as light is turned on again.

In light of the weak observed dynamics, and the fact that in common geophysical applications relaxation times can vary over multiple orders of magnitude (e.g., Pelton et al. 1978; Weller et al. 2016), the question arises whether the small variations in the relaxation time are reliable. One argument providing growth light turned off. Selected time spans are enlarged in the lower row (b-d)

confidence in the results is the large number of consistent measurements over multiple day-night cycles that comprise the time series of $\tau_{\text {mean }}$ in Fig. 12 . This indicates that the underlying signal is stronger than the noise components. However, in order to test whether the frequency sampling used in this experiment is suitable to represent such small changes in $\tau_{\text {mean }}$, a simple synthetic study was conducted: 10 EIS spectra with peaks corresponding to relaxation times between $16 \mathrm{~ms}$ and $20 \mathrm{~ms}$, representing the minimal and maximal relaxation times recovered in the experiment, were modeled and subsequently analyzed with the DD procedure (Fig. 13a). Recovered $\tau_{\text {mean }}$ values show that the relaxation times are still distinguishable (Fig. 13b). Note that the exact recovery (1:1) of the relaxation times underlying the original spectra is not expected due to the small differences in the used forward model (Cole-Cole) and the inverse model (Debye model) (e.g., Weigand and Kemna 2016b).

\section{Interpretation of diurnal patterns}

Plants regulate physiological activity in feedback loops tied to the circadian clock (e.g., Greenham and McClung 2015) and root systems can exhibit systematic variations in their hydraulic conductivity, linked to the diurnal light cycle (e.g., Parsons and Kramer 1974; 
a)
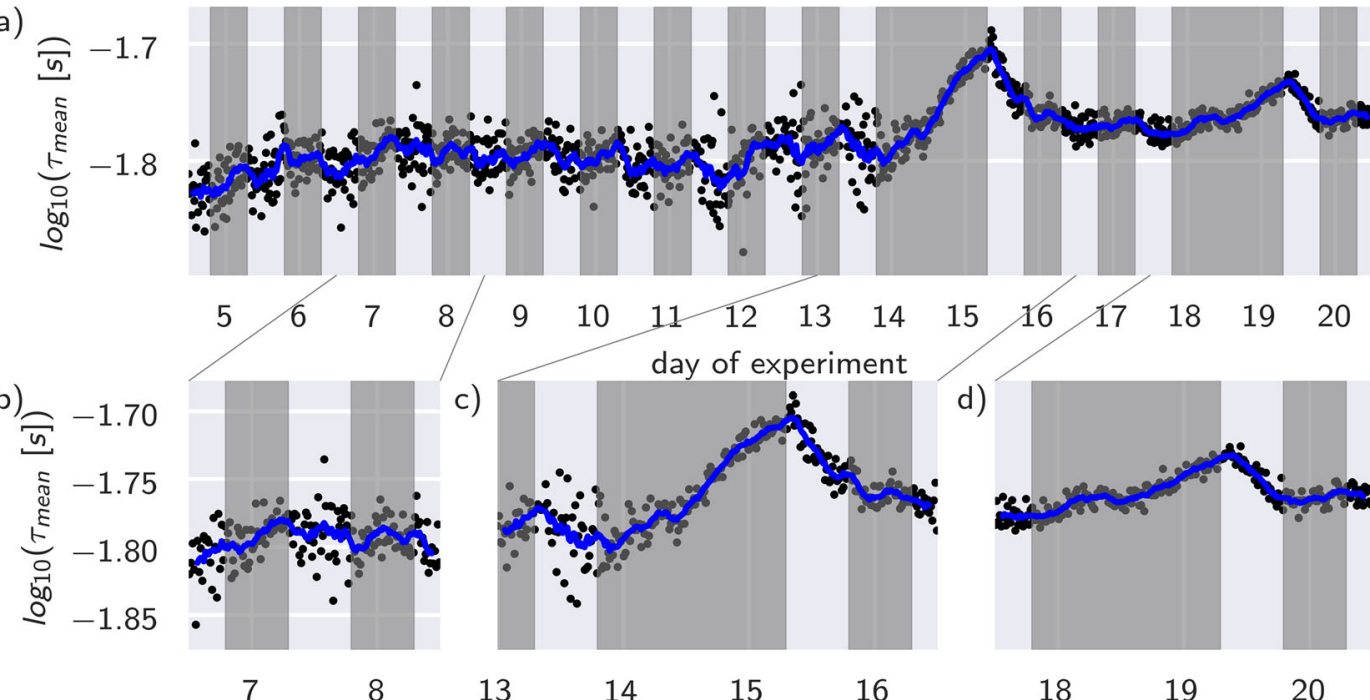

$\begin{array}{llll}11 & 12 & 13 & 14\end{array}$

15

day of experiment

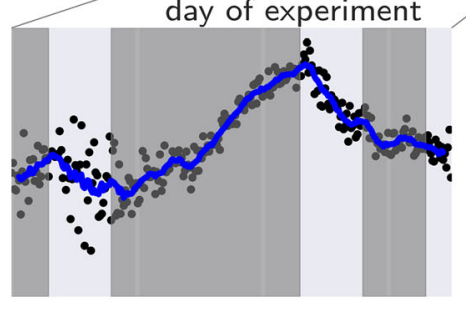

15

16

day of experiment

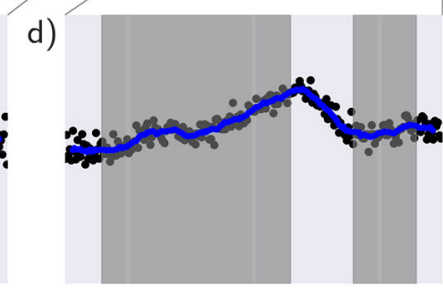

$18 \quad 19 \quad 20$

day of experiment
Fig. 12 Time series of the parameter $\tau_{\text {mean }}$ (black), as recovered from the DD of the EIS measurements on the root system. Shaded regions indicate time spans in dark conditions, i.e., with the growth light turned off. Blue curve in a) and b): Moving average filter applied to the time-series (window size of 11, ca. 70 min interval). Selected time spans are enlarged in the lower row (b-d)
Carvajal et al. 1996; Clarkson et al. 2000). Similarly, nutrient uptake also follows light/dark cycles, with reduced uptake (e.g., Delhon et al. 1995; Carvajal et al. 1996) and nutrient accumulation in the roots at night (e.g., Delhon et al. 1995). Interestingly, these diurnal cycles can be disturbed and changed by abnormal light patterns (e.g., Delhon et al. 1995; Carvajal et al. 1996). Relating to this experiment, we conclude that physiological processes in the root system are the most likely cause for the observed variations of the electrical properties (Figs. 10 - 12, supplement Figure S4) and we propose the following, simplified interpretation.

Electrical conduction across and within the root system depends on the electrical conduction properties of the aqueous solutions and membranes within the root system (assuming no significant changes in the conductivity of the bathing solution). Increased water/solute uptake during daylight hours goes along
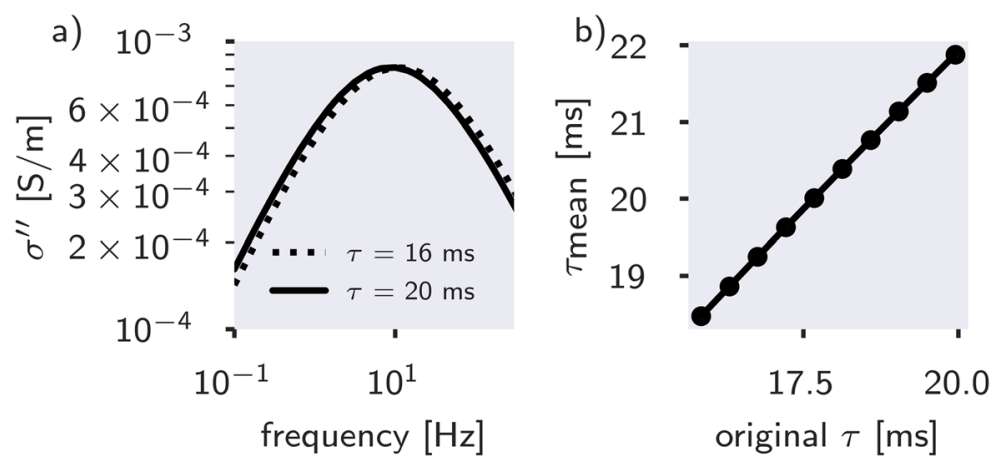

Fig. 13 a $\sigma^{\prime \prime}$ for two synthetically generated EIS signatures with slightly shifted polarization peaks determined by a difference in relaxation times of only $4 \mathrm{~ms}$. The signatures were generated with the Cole-Cole model (e.g., Pelton et al. 1978),

and the same frequencies were modeled as used in experiment 3. b Characteristic relaxation times recovered from the Debye decomposition of 10 spectra with peaks linearly distributed between the spectra shown in a) 
with increased transmissivity of root membranes for ions and thus increased electric current flow due to an external electric field in these periods, manifesting as an increased overall conductivity, measured in terms of $\sigma_{0}^{\mathrm{a}}$ (Fig. 10). Correspondingly, at times of low water/solute fluxes across root membranes, these are electrically more resistive, reflected by decreased $\sigma_{0}^{\mathrm{a}}$ values.

We assume that the electrical polarization properties of the root system, measured in terms of $m_{\text {tot }}^{\text {n, a }}$ and $\tau_{\text {mean }}$, are controlled by the polarizability of EDLs forming at root membranes. Increased nutrient uptake during daylight hours goes along with increased ion fluxes across root membranes. These affect the trans-membrane electric potential difference and thus the strength of the EDLs (i.e. diffuse layer thickness) at the membranes (e.g., Kinraide et al. 1998). More pronounced EDLs in turn lead to stronger EDL polarization in an external electric field. Therefore the decrease of $m_{\text {tot }}^{\mathrm{n}, \mathrm{a}}$ during daylight hours and its increase during periods of darkness (Fig. 11) suggest that the increased ion fluxes across root membranes due to increased water/nutrient uptake lead to a weakening of the EDLs at the membranes by lowering the trans-membrane electric potential difference. Such a mechanism would be similar to the weakening of EDLs around metallic particles, immersed in an electrolyte solution, through charge transfer reactions at the electrolyte-metal interface (e.g., Bücker et al. 2018).

The observed, relatively weak variation of $\tau_{\text {mean }}$ (Fig. 12) suggests that also the average length scale of the EDL polarization processes varies slightly over the day-night cycles. Here an increase of $\tau_{\text {mean }}$ correlates roughly with an increase of $m_{\text {tot }}^{\text {n, a }}$ during night time conditions, while both decrease during daylight hours (supplement Fig. S4). More pronounced EDLs (larger $\left.m_{\text {tot }}^{\text {n,a }}\right)$ are characterized by spatially more extended diffuse layers, which might be related to longer ion relaxation pathways upon polarization and therefore might explain increased values of $\tau_{\text {mean }}$. The quantification of the polarization length scale, as suggested by the observed $\tau_{\text {mean }}$ values, is addressed in the Discussion section.

While $m_{\text {tot }}^{\mathrm{n}, \mathrm{a}}$ and $\tau_{\text {mean }}$ are roughly correlated, their variation is not perfectly synchronous but $\tau_{\text {mean }}$ values lag somewhat behind $m_{\text {tot }}^{\text {n,a }}$ values during dark hours (see supplement Fig. S4 for an overlay of the temporal evolution of the DD parameters). We do not attempt to explain this phenomenon; however, we note that this behavior is similar to patterns observed for hydraulic conductance and ion concentrations in cotton plants over diurnal cycles (e.g., Parsons and Kramer 1974). Changes in electrical parameters that occur prior to changes in the light environment could indicate saturation of certain uptake/translocation processes, and thus provide additional information on the internal physiological state (rather than just mirroring external environmental factors such as light intensity).

\section{Discussion}

\section{Electrical polarization of roots}

The three experiments presented in this study demonstrate that sEIT and EIS are capable of capturing changes in electrical parameters (conductivity, chargeability, relaxation time) caused by internal physiological processes within crop root systems. This directly confirms that the root electrical polarization response is sensitive to root physiological processes (hypothesis 3 ) and that the signatures can be monitored with sEIT/EIS (hypothesis 4).

Our first hypothesis, that total chargeability is related to overall surface area comprised by EDLs and thus also to root system volume, is indirectly supported by the findings of experiment 1 . Here we observed highest polarization magnitudes near the stem area, where obviously most biomatter is present (cf. Fig. 2a).

Experiments 2 and 3 show consistent changes of the average relaxation time due to external environmental factors that affect certain physiological states within the root systems. Following our second hypothesis, the relaxation time is related to the length scale at which the polarization processes take place. At this stage, we do not have knowledge about the actual underlying polarization mechanisms and their length scale. However, based on the assumption that the polarization is of electrochemical nature, involving electromigrative and diffusive ion fluxes within or in the vicinity of EDLs forming at root cell membranes, it is plausible to postulate a general relationship between relaxation time $\tau$ and length scale $\Lambda$ according to

$$
\tau=\frac{\Lambda^{2}}{2 D}
$$


as typical for diffusion-controlled processes and as established for electrochemical polarization around electrically charged particles (e.g., Schurr 1964; Revil and Florsch 2010; Bücker et al. 2018) or bacteria (Revil et al. 2012; Mellage et al. 2018). In Eq. 6, $D$ denotes the diffusion coefficient, which for ions in aqueous solutions typically ranges between 0.5 . $10^{-9} \mathrm{~m}^{2} / \mathrm{s}$ and $2 \cdot 10^{-9} \mathrm{~m}^{2} / \mathrm{s}$ (Nobel 2009).

Average relaxation time values observed in our experiments range from 5 to $16 \mathrm{~ms}$ for experiment 1 (Fig. 3), from 3 to $10 \mathrm{~ms}$ for experiment 2 (Fig. 8), and from 16 to $20 \mathrm{~ms}$ for experiment 3 (Fig. 12), that is, they all have the same order of magnitude. Assuming a diffusion coefficient of $D=10^{-9} \mathrm{~m}^{2} / \mathrm{s}$ for the ions involved in the EDL polarization processes, the relaxation time range 3-20 ms translates into length scales between 2 and $7 \mu \mathrm{m}$ using Eq. 6 . We note that these values represent rough estimates only and should be interpreted with caution. Neither the validity of a relationship according to Eq. 6 has been proven yet for polarization processes in roots, nor the assumed value for the diffusion coefficient must be accurate. In fact, the diffusion coefficient can be expected to be smaller within the cell walls and membranes than in the unrestricted free electrolyte, which would result in smaller length scales than the values stated above. However, the obtained length scale estimates suggest that, for the root systems investigated here, the polarization processes take place at the micrometer scale (or below), that is, within single root or even fine root segments.

We believe that a better understanding of the mechanistic origin of the polarization processes, and the involved length scales, can be gained by coupling electrochemical polarization models established for porous/granular media (see Kemna et al. (2012) and, for more recent work, Bücker et al. (2018) and references therein) with existing conceptual models to describe water and ion fluxes in root elements (e.g., Foster and Miklavcic 2014, 2016; Couvreur et al. 2017; Meunier et al. 2017). This way chemical properties such as diffusion coefficients, ion concentrations and mobilities of different structures within roots can be taken into account in an electrodynamic modeling context.

The systematic variation of electrical properties of root systems tied to light cycles suggests new fields of application for spectroscopic and tomographic electrical impedance measurements. EIS and sEIT could develop into useful companion tools for the study of processes driven by circadian clocks and other exogenous stimuli, for example, and could be helpful in transferring results from controlled laboratory experiments to the field scale, where sEIT/EIS are likewise applicable.

\section{Methodological considerations for future experimental studies}

In order to better characterize the physiological processes underlying root electrical responses, in future studies care should be taken to control and monitor the environmental conditions (e.g., temperature, humidity, light conditions) during the experiments. Moreover, attempts should be made to increase the number of experimental repetitions to consolidate the results and their biophysical interpretation (see, e.g., Fig. 5 in Thomas et al. 2016). While this has been achieved already for studies involving two-electrode capacitance measurements (e.g., Cseresnyés et al. 2016) and four-electrode SIP/EIS measurements (e.g., Postic and Doussan 2016), it is challenging for tomographic, broadband four-electrode impedance measurements given the data acquisition and processing demands. Spatial resolution of computed sEIT images might be increased by optimizing electrode locations in relation to the known or expected position of root systems, or specific regions of interest (e.g., Wagner et al. 2015), as well as by employing optimized measurement configurations (e.g., Stummer et al. 2004; Wilkinson et al. 2006; al Hagrey and Petersen 2011).

After the successful application of sEIT and EIS for the monitoring of roots in aqueous solutions in this study, in future experiments also substrates and soils need to be incorporated with a view to field applications, making sEIT measurements and their interpretation more complicated (e.g., Weigand 2017). One of the most challenging aspects here is the variable water content of the soil, in particular in the root zone, which has a strong influence on its electrical polarization properties (phase values, $\sigma^{\prime \prime}$ ) (e.g., Ghorbani et al. 2008; Breede et al. 2012; Kelter et al. 2018). Absolute signal strengths, as well as signal dynamics, of the soil may be of similar magnitude, or even larger, than the signatures from the root system (compare, for example, $\sigma^{\prime \prime}$ values in Fig. 2c with $\sigma^{\prime \prime}$ values reported in Ghorbani et al. 2008). Since both soil and root electrical signatures inevitably overlap in the measurements, 
the extraction of the root response will require to take the soil response and its dynamics due to soil water variations properly into account (e.g., Cseresnyés et al. 2016; Weigand and Kemna 2017; Weigand 2017, and references therein). Here, independent estimates of soil water content can be obtained from DC (directcurrent) resistivity measurements based on established pedophysical relationships (e.g., Werban et al. 2008; Srayeddin and Doussan 2009; al Hagrey and Petersen 2011).

In light of these challenges involved in applying sEIT to more complex, realistic root-soil scenarios, we believe that improved soil-root electrical models will be essential to differentiate between the various contributions in measured spectral signatures. For example models that simulate root structure and growth, including water uptake and redistribution processes in the soil-root continuum (e.g., Javaux et al. 2008; Leitner et al. 2014; Postma et al. 2017), could be coupled with novel electrochemical polarization models for individual root elements (see discussion in subsection Interpretation of diurnal patterns) to improve our process understanding.

\section{Conclusions}

In this study we showed that electrical polarization measurements are sensitive to both root structure and physiological state. We used spectral electrical impedance tomography (sEIT) to image crop root systems in aqueous solutions based on their polarization response, as well as high-speed electrical spectroscopic measurements to observe systematic changes over the diurnal cycle of a plant-root system. The frequency dependence of the electrical response provides additional information about the root system, and estimates using established models for the electrochemical polarization around mineral particles or bacteria suggest that the observed polarization processes take place on the $\mu \mathrm{m}$ scale, i.e., well within single root elements. Both signal strength and spectral characteristics varied during the monitoring of two oilseed root systems in a nutrient-deprived aqueous environment, highlighting the ability of sEIT to image physiological changes within root systems. Spectral signatures measured with a high temporal sampling rate showed that diurnal patterns associated with light and dark conditions can be captured using electrical polarization measurements, again indicating their sensitivity toward the inner physiological functioning of root systems. This directly points to possible applications with regard to investigating circadian clocks and associated processes. Future work should be directed towards proving the suitability of the sEIT method to characterize root systems also embedded in soil or substrate, which is required for the use at the field scale and complicated by the overlapping of soil and root electrical responses, as well as towards the development of improved soil-root physicochemical models for a better process and electrical signature understanding.

Acknowledgements We are grateful to Egon Zimmermann for valuable discussions regarding the measurement setup and procedures, and we thank Michael Stamm for his huge help in conducting the third experiment.

Parts of this research were funded by the Deutsche Forschungsgemeinschaft (DFG) in the framework of the project "Non-destructive characterization and monitoring of root structure and function at the rhizotron and field scale using spectral electrical impedance tomography" (KE 1138/1-1) and the collaborative research centre "Patterns in soil-vegetationatmosphere systems: monitoring, modeling and data assimilation" (SFB/TR 32).

We are grateful to Florian Wagner for a thorough presubmission review, as well as for the constructive comments of two anonymous reviewers.

Data Availability Measurement data and analysis scripts are available under the https://doi.org/10.5281/zenodo.1320755

Open Access This article is distributed under the terms of the Creative Commons Attribution 4.0 International License (http:// creativecommons.org/licenses/by/4.0/), which permits unrestricted use, distribution, and reproduction in any medium, provided you give appropriate credit to the original author(s) and the source, provide a link to the Creative Commons license, and indicate if changes were made.

\section{References}

Amato M, Basso B, Celano G, Bitella G, Morelli G, Rossi $\mathrm{R}$ (2008) In situ detection of tree root distribution and biomass by multi-electrode resistivity imaging. Tree Physiol 28(10):1441-1448. https://doi.org/10.1093/treephys/28. 10.1441

Amato M, Bitella G, Rossi R, Gómez JA, Lovelli S, Gomes JJF (2009) Multi-electrode 3D resistivity imaging of alfalfa root zone. Eur J Agron 31(4):213-222. https://doi.org/10.1016/j. eja.2009.08.005

Archie G (1942) The electrical resistivity log as an aid in determining some reservoir characteristics. Petroleum Technology 54-62. https://doi.org/10.2118/942054-G 
Bačić G, Ratković S (1987) NMR Studies of radial exchange and distribution of water in maize roots: the relevance of modelling of exchange kinetics. J Exp Bot 38(8):12841297. https://doi.org/10.1093/jxb/38.8.1284

Barsoukov E, Macdonald J (eds) (2005) Impedance spectroscopy: theory, experiment, and applications. WileyInterscience, Hoboken. https://doi.org/10.1002/0471716243

Binley A, Kemna A (2005) DC resistivity and induced polarization methods. In: Rubin Y, Hubbard SS (eds) Hydrogeophysics. Springer, Netherlands, pp 129-156. https://doi.org/10.1007/1-4020-3102-5_5

Breede K, Kemna A, Esser O, Zimmermann E, Vereecken H, Huisman J (2012) Spectral induced polarization measurements on variably saturated sand-clay mixtures. Near Surface Geophysics 10(6):479-489. https://doi.org/10.3997/18 73-0604.2012048

Bücker M, Flores Orozco A, Kemna A (2018) Electro-chemical polarization around metallic particles-part 1: the role of diffuse-layer and volume-diffusion relaxation. Geophysics 83(4):1-53. https://doi.org/10.1190/geo2017-0401.1

Cabañero FJ, Carvajal M (2007) Different cation stresses affect specifically osmotic root hydraulic conductance, involving aquaporins, ATPase and xylem loading of ions in Capsicum annuum, 1. plants. J Plant Physiol 164(10):1300-1310. https://doi.org/10.1016/j.jplph.2006.08.010

Carminati A, Moradi A, Vetterlein D, Vontobel P, Lehmann E, Weller U, Vogel H, Oswald S (2010) Dynamics of soil water content in the rhizosphere. Plant Soil 332(1):163176. https://doi.org/10.1007/s11104-010-0283-8

Carvajal M, Cooke D, Clarkson D (1996) Responses of wheat plants to nutrient deprivation may involve the regulation of water-channel function. Planta 199(3):372-381. https://doi. org/10.1007/BF00195729

Chloupek O (1972) The relationship between electric capacitance and some other parameters of plant roots. Biol Plant 14(3):227-230. https://doi.org/10.1007/BF02921255

Chloupek O (1976) Die Bewertung des Wurzelsystems von Senfpflanzen auf Grund der dielektrischen Eigenschaften und mit Rücksicht auf den Endertrag. Biol Plant 18(November):44-49. https://doi.org/10.1007/BF02922333

Clarkson D, Carvajal M, Henzler T, Waterhouse R, Smyth A, Cooke D, Steudle E (2000) Root hydraulic conductance: diurnal aquaporin expression and the effects of nutrient stress. J Exp Bot 51(342):61-70. https://doi.org/10.1093/ jexbot/51.342.61

Couvreur V, Faget M, Lobet G, Javaux M, Chaumont F, Draye $X$ (2017) Novel multiscale insights into the composite nature of water transport in roots. bioRxiv. https://doi.org/ 10.1101/147314

Cramer M, Hawkins H, Verboom G (2009) The importance of nutritional regulation of plant water flux. Oecologia 161(1):15-24. https://doi.org/10.1007/s00442-0091364-3

Cseresnyés I, Tünde T, Végh K, Anton A, Rajkai K (2013) Electrical impedance and capacitance method: a new approach for detection of functional aspects of arbuscular mycorrhizal colonization in maize. Eur J Soil Biol 54:25-31. https://doi.org/10.1016/j.ejsobi.2012.11.001

Cseresnyés I, Takács T, Füzy A, Rajkai K (2014) Simultaneous monitoring of electrical capacitance and water uptake activity of plant root system. Int Agrophys 28(4):537-541. https://doi.org/10.2478/intag-2014-0044

Cseresnyés I, Rajkai K, Takács T (2016) Indirect monitoring of root activity in soybean cultivars under contrasting moisture regimes by measuring electrical capacitance. Acta Physiol Plant 38(5):121. https://doi.org/10.1007/s11738-016-2149z

Dalton F (1995) In-situ root extent measurements by electrical capacitance methods. Plant Soil 173(1):157-165. https://doi. org/10.1007/BF00155527

De Donno G, Cardarelli E (2014) 3D complex resistivity tomography on cylindrical models using EIDORS. Near Surface Geophysics 12(5):587-598. https://doi.org/10.3997/18730604.2014016

Delhon P, Gojon A, Tillard P, Passama L (1995) Diurnal regulation of NO3- uptake in soybean plants I. Changes in NO3- influx, efflux, and $\mathrm{N}$ utilization in the plant during the day/night cycle. J Exp Bot 46(10):1585-1594. https://doi. org/10.1093/jxb/46.10.1585

Dentith M, Mudge S (2014) Geophysics for the mineral exploration geoscientist. Cambridge University Press, Cambridge

Ellis T, Murray W, Kavalieris L (2013) Electrical capacitance of bean (Vicia faba) root systems was related to tissue density - a test for the Dalton Model. Plant and soil 366(12):575-584. https://doi.org/10.1007/s11104-012-1424-z

Everett M (2013) Near-surface applied geophysics. Cambridge University Press. https://doi.org/10.1007/s11001-014-921 8-8

Foster K, Miklavcic S (2014) On the competitive uptake and transport of ions through differentiated root tissues. J Theor Biol 340:1-10. https://doi.org/10.1016/j.jtbi.2013.09.004

Foster K, Miklavcic S (2016) Modeling root zone effects on preferred pathways for the passive transport of ions and water in plant roots. Front Plant Sci 7:914. https://doi.org/10.3389/ fpls.2016.00914

Gaiser T, Perkons U, Küpper P, Puschmann D, Peth S, Kautz T, Pfeifer J, Ewert F, Horn R, Köpke U (2012) Evidence of improved water uptake from subsoil by spring wheat following lucerne in a temperate humid climate. Field Crop Res 126:56-62. https://doi.org/10.1016/j.fcr.2011.09.019

Ghorbani A, Cosenza P, Ruy S, Doussan C, Florsch N (2008) Non-invasive monitoring of water infiltration in a silty clay loam soil using spectral induced polarization. Water Resour Res 44(8):1-18. https://doi.org/10.1029/2007WR006114

Greenham K, McClung C (2015) Integrating circadian dynamics with physiological processes in plants. Nat Rev Genet 16(10):598-610. https://doi.org/10.1038/nrg3976

Gregory P, Hutchison D, Read D, Jenneson P, Gilboy W, Morton E (2003) Non-invasive imaging of roots with high resolution X-ray micro-tomography. In: Roots: the dynamic interface between plants and the Earth. Springer, pp 351359. https://doi.org/10.1023/A:1026179919689

Günther T, Rücker C, Spitzer K (2006) Three-dimensional modelling and inversion of DC resistivity data incorporating topography - II. inversion. Geophys J Int 166(2):506-517. https://doi.org/10.1111/j.1365-246X.2006.03011.x

al Hagrey S, Petersen T (2011) Numerical and experimental mapping of small root zones using optimized surface and borehole resistivity tomography. Geophysics 76(2):G25G35. https://doi.org/10.1190/1.3545067 
Heřmanská A, Středa T, Chloupek O (2015) Improved wheat grain yield by a new method of root selection. Agron Sustain Dev 35(1):195-202. https://doi.org/10.1007/s13593014-0227-4

Javaux M, Schröder T, Vanderborght J, Vereecken H (2008) Use of a three-dimensional detailed modeling approach for predicting root water uptake. Vadose Zone J 7(3):1079-1088. https://doi.org/10.2136/vzj2007.0115

Kelter M, Huisman J, Zimmermann E, Vereecken H (2018) Field evaluation of broadband spectral electrical imaging for soil and aquifer characterization. J Appl Geophys 159:484 496. https://doi.org/10.1016/j.jappgeo.2018.09.029

Kemna A (2000) Tomographic inversion of complex resistivity - theory and application. $\mathrm{PhD}$ thesis, Ruhr-Universität Bochum. https://doi.org/10.1111/1365-2478.12013

Kemna A, Binley A, Cassiani G, Niederleithinger E, Revil A, Slater L, Williams K, Flores Orozco A, Haegel F, Hoerdt A, Kruschwitz S, Leroux V, Titov K, Zimermann E (2012) An overview of the spectral induced polarization method for near-surface applications. Near Surface Geophysics 10(6):453-468. https://doi.org/10.3997/1873-0604.2012027

Kinraide T, Wang P (2010) The surface charge density of plant cell membranes $(\sigma)$ : an attempt to resolve conflicting values for intrinsic $\sigma$. J Exp Bot 61:2507-2518. https://doi.org/10. 1093/jxb/erq082

Kinraide T, Yermiyahu U, Rytwo G (1998) Computation of surface electrical potentials of plant cell membranes correspondence to published zeta potentials from diverse plant sources. Plant Physiol 118(2):505-512. https://doi.org/10. 1104/pp.118.2.505

LaBrecque D, Miletto M, Daily W, Ramirez A, Owen E (1996) The effects of noise on Occam's inversion of resistivity tomography data. Geophysics 61(2):538-538. https://doi.org/10.1190/1.1443980

van der Leij M, Smith S, Miller A (1998) Remobilisation of vacuolar stored nitrate in barley root cells. Planta 205(1):64 72. https://doi.org/10.1007/s004250050297

Leitner D, Meunier F, Bodner G, Javaux M, Schnepf A (2014) Impact of contrasted maize root traits at flowering on water stress tolerance - a simulation study. Field Crop Res 165:125-137. https://doi.org/10.1016/j.fcr.2014.05.009, crop root system behaviour and yield

Liao A, Zhou Q, Zhang Y (2015) Application of 3D electrical capacitance tomography in probing anomalous blocks in water. J Appl Geophys 117:91-103. https://doi.org/10.1016/ j.jappgeo.2015.03.030

Liu X, Dong X, Xue Q, Leskovar D, Jifon J, Butnor J, Marek $\mathrm{T}$ (2017) Ground penetrating radar (GPR) detects fine roots of agricultural crops in the field. Plant Soil, 1-15. https://doi.org/10.1007/s11104-017-3531-3

Lyklema J (2005) Fundamentals of interface and colloid science: solid-liquid interfaces, vol 2. Academic press, Cambridge

Lyklema J, Dukhin S, Shilov V (1983) The relaxation of the double layer around colloidal particles and the lowfrequency dielectric dispersion: Part I. Theoretical considerations. J. Electroanal Chem Interfacial Electrochem 143(1):1-21. https://doi.org/10.1016/S0022-0728(83)8025 $1-4$
Mancuso S (2012) Measuring roots: an updated approach. Springer Science \& Business Media, iSBN: 978-3-64222067-8

Mary B, Peruzzo L, Boaga J, Schmutz M, Wu Y, Hubbard SS, Cassiani G (2018) Small scale characterization of vine plant root water uptake via $3 \mathrm{~d}$ electrical resistivity tomography and mise-à-la-masse method. Hydrol Earth Syst Sci 2018:5427-5444. https://doi.org/10.5194/ hess-22-5427-2018

Mellage A, Smeaton C, Furman A, Atekwana E, Rezanezhad F, Van Cappellen P (2018) Linking spectral induced polarization (SIP) and subsurface microbial processes: Results from sand column incubation experiments. Environmental science \& technology. https://doi.org/10.1021/acs.est. $7 \mathrm{~b} 04420$

Metzner R, Eggert A, van Dusschoten D, Pflugfelder D, Gerth S, Schurr U, Uhlmann N, Jahnke S (2015) Direct comparison of MRI and X-ray CT technologies for 3D imaging of root systems in soil: potential and challenges for root trait quantification. Plant Methods 11(1):1. https://doi.org/10. 1186/s13007-015-0060-Z

Meunier F, Draye X, Vanderborght J, Javaux M, Couvreur V (2017) A hybrid analytical-numerical method for solving water flow equations in root hydraulic architectures. Appl Math Model 52:648-663. https://doi.org/10.1016/j.apm. 2017.08.011

Moradi A, Conesa H, Robinson B, Lehmann E, Kuehne G, Kaestner A, Oswald S, Schulin R (2009) Neutron radiography as a tool for revealing root development in soil: capabilities and limitations. Plant Soil 318(1-2):243-255. https://doi.org/10.1007/s11104-008-9834-7

Newill P, Karadaglić D, Podd F, Grieve B, York TA (2014) Electrical impedance imaging of water distribution in the root zone. Meas Sci Technol 25(5):055110. https://doi.org/10.1088/0957-0233/25/5/055110

Nobel P (2009) Physicochemical and environmental plant physiology, 4th edn. Cambridge, Academic Press

Nordsiek S, Weller A (2008) A new approach to fitting induced-polarization spectra. Geophysics 73(6):F235F245. https://doi.org/10.1190/1.2987412

Oberdörster C, Vanderborght J, Kemna A, Vereecken H (2010) Investigating preferential flow processes in a forest soil using time domain reflectometry and electrical resistivity tomography. Vadose Zone J 9(2):350-361. https://doi.org/10.2136/vzj2009.0073

Ozier-Lafontaine H, Bajazet T (2005) Analysis of root growth by impedance spectroscopy (EIS). Plant Soil 277(1-2):299_ 313. https://doi.org/10.1007/s11104-005-7531-3

Parsons L, Kramer P (1974) Diurnal cycling in root resistance to water movement. Physiologia Plantarum 30(1):19-23. https://doi.org/10.1111/j.1399-3054.1974.tb04985.x

Pelton W, Ward S, Hallof P, Sill W, Nelson P (1978) Mineral discrimination and removal of inductive coupling with multifrequency IP. Geophysics 43(3):588-609. https://doi.org/10.1190/1.1440839

Postic F, Doussan C (2016) Benchmarking electrical methods for rapid estimation of root biomass. Plant Methods 12(1):33. https://doi.org/10.1186/s13007-016-0133-7

Postma J, Kuppe C, Owen M, Mellor N, Griffiths M, Bennett M, Lynch J, Watt M (2017) OpenSimRoot: widening the scope and application of root architectural models. New 
Phytologist 215(3):1274-1286. https://doi.org/10.1111/ nph.14641,2017-24197

Repo T, Korhonen A, Laukkanen M, Lehto T, Silvennoinen R (2014) Detecting mycorrhizal colonisation in Scots pine roots using electrical impedance spectra. Biosyst Eng 121:139-149. https://doi.org/10.1016/j.biosystemseng. 2014.02.014

Revil A, Florsch N (2010) Determination of permeability from spectral induced polarization in granular media. Geophys J Int 181(3):1480-1498. https://doi.org/10.1111/j.1365246X.2010.04573.X

Revil A, Atekwana E, Zhang C, Jardani A, Smith S (2012) A new model for the spectral induced polarization signature of bacterial growth in porous media. Water Resour Res 48(9). https://doi.org/10.1029/2012WR011965

Revil A, Eppehimer J, Skold M, Karaoulis M, Godinez L, Prasad M (2013) Low-frequency complex conductivity of sandy and clayey materials. J Colloid Interface Sci 398:193-209. https://doi.org/10.1016/j.jcis.2013.01.015

Rhoades J, Manteghi N, Shouse P, Alves W (1989) Soil electrical conductivity and soil salinity: new formulations and calibrations. Soil Sci Soc Am J 53(2):433-439. https://doi.org/ 10.2136/sssaj1989.03615995005300020020x

Roose T, Keyes S, Daly K, Carminati A, Otten W, Vetterlein D, Peth S (2016) Challenges in imaging and predictive modeling of rhizosphere processes. Plant Soil, 1-30. https://doi.org/10.1007/s11104-016-2872-7

Schurr J (1964) On the theory of the dielectric dispersion of spherical colloidal particles in electrolyte solution. J Phys Chem 68(9):2407-2413. https://doi.org/10.1021/j100791 a004

Schwan H (1957) Electrical properties of tissue and cell suspensions. Advances in Biological and Medical Physics, vol 5, Elsevier, 147-209. https://doi.org/10.1016/B978-1-48323111-2.50008-0

Schwarz G (1962) A theory of the low-frequency dielectric dispersion of colloidal particles in electrolyte solution. J Phys Chem 66(12):2636-2642. https://doi.org/10.1021/j100818 a067

Sen P, Goode P (1992) Influence of temperature on electrical conductivity on shaly sands. Geophysics 57(1):89-96. https://doi.org/10.1190/1.1443191

Srayeddin I, Doussan C (2009) Estimation of the spatial variability of root water uptake of maize and sorghum at the field scale by electrical resistivity tomography. Plant and soil 319(1-2):185-207. https://doi.org/10.1007/s11104008-9860-5

Stummer P, Maurer H, Green A (2004) Experimental design: electrical resistivity data sets that provide optimum subsurface information. Geophysics 69(1):120-139. https://doi.org/10.1190/1.1649381

Sumner J (1976) Principles of induced polarization for geophysical exploration. Springer, Berlin. https://doi.org/10.1111/j. 1365-246X.1977.tb04186.x

Tarasov A, Titov K (2013) On the use of the Cole-Cole equations in spectral induced polarization. Geophys $\mathrm{J}$ Int 195(1):352-356. https://doi.org/10.1093/gji/ggt251

Telford W, Geldart LP, Sheriff R (1990) Applied geophysics, vol 1. Cambridge University Press, Cambridge
Thomas C, Graham N, Hayden R, Meacham M, Neugebauer K, Nightingale M, Dupuy L, Hammond J, White P, Broadley M (2016) High-throughput phenotyping (HTP) identifies seedling root traits linked to variation in seed yield and nutrient capture in field-grown oilseed rape (Brassica napus 1.) Ann Bot 118(4):655-665. https://doi.org/10.1093/aob/ mew046

Tinker P, Nye P (2000) Solute movement in the rhizosphere. Oxford University Press, Inc., New York. https://doi.org/10. 1046/j.1365-2389.2001.00418-2.x

Wagner FM, Günther T, Schmidt-Hattenberger C, Maurer $\mathrm{H}$ (2015) Constructive optimization of electrode locations for target-focused resistivity monitoring. Geophysics 80(2):E29-E40. https://doi.org/10.1190/geo2014-0214.1

Weigand M (2017) Monitoring structural and physiological properties of crop roots using spectral electrical impedance tomography. University of Bonn, PhD thesis. https://doi.org/10.5281/zenodo.400833

Weigand M, Kemna A (2016a) Debye decomposition of time-lapse spectral induced polarisation data. Comput Geosci 86:34-45. https://doi.org/10.1016/j.cageo.2015.09. 021

Weigand M, Kemna A (2016b) Relationship between Cole-Cole model parameters and spectral decomposition parameters derived from SIP data. Geophys J Int 205:1414-1419. https://doi.org/10.1093/gji/ggw099

Weigand M, Kemna A (2017) Multi-frequency electrical impedance tomography as a non-invasive tool to characterize and monitor crop root systems. Biogeosciences 14(4):921-939. https://doi.org/10.5194/bg-14-921-2017

Weigand M, Flores Orozco A, Kemna A (2017) Reconstruction quality of SIP parameters in multi-frequency complex resistivity imaging. Near Surface Geophysics 15:187-199. https://doi.org/10.3997/1873-0604.2016050

Weller A, Zhang Z, Slater L, Kruschwitz S, Halisch M (2016) Induced polarization and pore radius - discussion. Geophysics 81(5):D519-D526. https://doi.org/10.1190/geo 2016-0135.1

Werban U, al Hagrey SA, Rabbel W (2008) Monitoring of root-zone water content in the laboratory by $2 \mathrm{D}$ geoelectrical tomography. J Plant Nutr Soil Sci 171(6):927-935. https://doi.org/10.1002/jpln.200700145

Whalley W, Binley A, Watts C, Shanahan P, Dodd I, Ober E, Ashton R, Webster C, White R, Hawkesford MJ (2017) Methods to estimate changes in soil water for phenotyping root activity in the field. Plant Soil 1-16. https://doi.org/10.1007/s11104-016-3161-1

White P, George T, Gregory P, Bengough A, Hallett P, McKenzie B (2013) Matching roots to their environment. Ann Bot 112(2):207-222. https://doi.org/10.1093/aob/mct123

Wilkinson P, Meldrum P, Chambers J, Kuras O, Ogilvy R (2006) Improved strategies for the automatic selection of optimized sets of electrical resistivity tomography measurement configurations. Geophys J Int 167(3):1119-1126. https://doi.org/10.1111/j.1365-246X.2006.03196.x

Wu W, Duncan R, Ma B (2017) Quantification of canola root morphological traits under heat and drought stresses with electrical measurements. Plant Soil 415(1):229-244. https://doi.org/10.1007/s11104-016-3155-z 
Zimmermann E, Kemna A, Berwix J, Glaas W, Vereecken H (2008) EIT measurement system with high phase accuracy for the imaging of spectral induced polarization properties of soils and sediments. Meas Sci Technol 19(9):094010. https://doi.org/10.1088/0957-0233/19/9/094010
Zimmermann E, Huisman J, Wolters B, van Waasen S (2012) Optimal electrode design for improved phase accuracy of spectral eit images. In: Proceedings of the 6th international symposcium on process tomography, Cape Town, South Africa, 26.03.2012-28.03.2012 